\title{
Testing gravity with galaxy-galaxy lensing and redshift-space distortions using CFHT-Stripe 82, CFHTLenS, and BOSS CMASS datasets ${ }^{\star}$
}

\author{
E. Jullo ${ }^{1}$, S. de la Torre ${ }^{1}$, M.-C. Cousinou ${ }^{2}$, S. Escoffier ${ }^{2}$, C. Giocoli ${ }^{3,4,5,6}$, R. B. Metcalf ${ }^{4,5}$, J. Comparat ${ }^{7}$, \\ H.-Y. Shan ${ }^{8}$, M. Makler ${ }^{9}$, J.-P. Kneib ${ }^{10,1}$, F. Prada ${ }^{11}$, G. Yepes ${ }^{12,13}$, and S. Gottlöber ${ }^{14}$ \\ 1 Aix-Marseille Univ., CNRS, CNES, LAM, Marseille, France \\ e-mail: eric.jullo@lam. fr \\ 2 Aix-Marseille Univ., CNRS/IN2P3, CPPM, Marseille, France \\ 3 Dipartimento di Fisica e Scienze della Terra, Università degli Studi di Ferrara, Via Saragat 1, 44122 Ferrara, Italy \\ 4 INAF - Osservatorio di Astrofisica e Scienza dello Spazio di Bologna, Via Gobetti 93/3, 40129 Bologna, Italy \\ 5 Dipartimento di Fisica e Astronomia, Alma Mater Studiorum Università di Bologna, Via Gobetti 93/2, 40129 Bologna, Italy \\ 6 INFN - Sezione di Bologna, Viale Berti Pichat 6/2, 40127 Bologna, Italy \\ 7 Max-Planck-Institut für extraterrestrische Physik, Giessenbachstrasse 1, 85748 Garching bei München, Germany \\ 8 Shanghai Astronomical Observatory (SHAO), Nandan Road 80, Shanghai 200030, PR China \\ 9 Centro Brasileiro de Pesquisas Físicas, Rio de Janeiro, RJ 22290-180, Brasil \\ 10 Institute of Physics, Laboratory of Astrophysics, Ecole Polytechnique Fédérale de Lausanne (EPFL), Observatoire de Sauverny, \\ 1290 Versoix, Switzerland \\ 11 Instituto de Astrofísica de Andalucía (CSIC), Glorieta de la Astronomía, 18080 Granada, Spain \\ 12 Departamento de Física Teórica, Módulo 15, Universidad Autónoma de Madrid, 28049 Madrid, Spain \\ 13 Centro de Investigación Avanzada en Física Fundamental (CIAFF), Universidad Autónoma de Madrid, 28049 Madrid, Spain \\ 14 Leibniz-Institut für Astrophysik (AIP), An der Sternwarte 16, 14482 Potsdam, Germany
}

Received 12 November 2018 / Accepted 2 May 2019

\begin{abstract}
The combination of galaxy-galaxy lensing (GGL) and redshift space distortion of galaxy clustering (RSD) is a privileged technique to test general relativity predictions and break degeneracies between the growth rate of structure parameter $f$ and the amplitude of the linear power spectrum $\sigma_{8}$. We performed a joint GGL and RSD analysis on 250 sq. deg using shape catalogues from CFHTLenS and CFHT-Stripe 82 and spectroscopic redshifts from the BOSS CMASS sample. We adjusted a model that includes non-linear biasing, RSD, and Alcock-Paczynski effects. We used an N-body simulation supplemented by an abundance matching prescription for CMASS galaxies to build a set of overlapping lensing and clustering mocks. Together with additional spectroscopic data, this helps us to quantify and correct several systematic errors, such as photometric redshifts. We find $f(z=0.57)=0.95 \pm 0.23, \sigma_{8}(z=$ $0.57)=0.55 \pm 0.07$ and $\Omega_{\mathrm{m}}=0.31 \pm 0.08$, in agreement with Planck cosmological results 2018 . We also estimate the probe of gravity $E_{\mathrm{G}}=0.43 \pm 0.10$, in agreement with $\Lambda \mathrm{CDM}-\mathrm{GR}$ predictions of $E_{\mathrm{G}}=0.40$. This analysis reveals that RSD efficiently decreases the GGL uncertainty on $\Omega_{\mathrm{m}}$ by a factor of 4 and by $30 \%$ on $\sigma_{8}$. We make our mock catalogues available on the Skies and Universe database.
\end{abstract}

Key words. cosmological parameters - cosmology: observations - large-scale structure of Universe

\section{Introduction}

Since its inception, general relativity theory (GR) has been constantly tested, starting with observations in the solar system and in our Galaxy (see e.g. Damour 2000). At cosmological scales, the advent of wide field survey experiments currently yields very high precision measurements in both the early and late ages of the universe. A Universe dominated by cold dark matter and a cosmological constant in the context of GR (hereafter $\Lambda \mathrm{CDM}-\mathrm{GR}$ model) reproduces all these observations with very high accuracy and for this reason, the model is often referred to as the standard reference model.

\footnotetext{
* The catalogues are available at the CDS via anonymous ftp to cdsarc.u-strasbg.fr $(130.79 .128 .5)$ or via http://cdsarc. u-strasbg.fr/viz-bin/qcat?]/A+A/627/A137 and at http:// www. skiesanduniverses.org
}

However, some slight tensions are emerging between predictions based on the cosmic microwave background measurements from the Planck mission at redshift $z=1089$ and measurements at redshifts $z<1$ obtained from galaxy clustering or gravitational lensing. In particular with Planck, the amplitude of the matter power spectrum $\sigma_{8}$ is larger and the Hubble constant $H_{0}$ is smaller than what is estimated at redshifts $z<1$ at about $2 \sigma$ confidence level (C.L.; Planck Collaboration XIII 2016; Beutler et al. 2014; Alam et al. 2017; Hildebrandt et al. 2017; DES Collaboration 2018). Although systematic errors in the analyses can explain a significant fraction of these discrepancies, they might nonetheless suggest some issues with our understanding and modelling of the expansion of the Universe, or of the large-scale structure formation probed by galaxy clustering and gravitational lensing.

The common approach to test $\Lambda \mathrm{CDM}-\mathrm{GR}$ at cosmological scales is either to measure the expansion history $H(z)$ 
of the Universe (e.g. Betoule et al. 2014; Alam et al. 2017; Magaña et al. 2015), or to measure the growth of structures traced by the velocity or density fields in redshift space (e.g. de la Torre et al. 2013; Tully et al. 2016; Martinet et al. 2018). In this paper, we combine galaxy-galaxy lensing (GGL) and RSD to test both aspects simultaneously at redshift $z=0.57$. The amplitude of GGL measurements is sensitive to $H(z)$ and the density field, whereas RSD probes the growth of structure $f(z)$ through galaxy peculiar velocities. The combination of these two observables has demonstrated its effectiveness at isolating the independent effects of the growth rate of structure $f(z)$, the amplitude of the matter power spectrum $\sigma_{8}$, and the dark energy equation of state parameter $w$ involved in $H(z)$ calculation (Simpson et al. 2013; de la Torre et al. 2017; Joudaki et al. 2017).

Zhang et al. (2007) proposed an alternative method to test deviations to GR. Assuming small scalar perturbations around the Friedmann-Lemaître-Robertson-Walker metric (FLRW) in the conformal Newtonian gauge $\mathrm{d} s^{2}=-a(\tau)^{2}[1+2 \Psi] \mathrm{d} \tau^{2}+$ $a(\tau)^{2}[1-2 \Phi] \mathrm{d} \mathbf{x}^{2}$, where $a$ is a scale factor, $\tau$ is the conformal time, and $\mathbf{x}$ are comoving coordinates, these authors proposed a statistics $E_{\mathrm{G}}$ sensitive to the gravitational slip between the two gravitational potentials $\Phi$ and $\Psi$ as follows:

$\left\langle E_{\mathrm{G}}\right\rangle=\left[\frac{\nabla^{2}(\Psi-\Phi)}{3 H_{0}^{2} a^{-1} f \delta}\right]$,

where all quantities are estimated at the redshift of interest. Reyes et al. (2010) proposed an associated observational estimator $E_{\mathrm{G}}=\Upsilon_{\mathrm{gm}} / \beta \Upsilon_{\mathrm{gg}}$ (see below in Sect. 6.4), which converges to $\left\langle E_{\mathrm{G}}\right\rangle$ in the large-scale limit where the galaxy bias $b$ and the distortion parameter $\beta=f / b$ converge to constant values. The small-scale filtered galaxy-matter cross-correlation $\Upsilon_{\mathrm{gm}}$ probed with GGL is sensitive to both $b$ and $\nabla^{2}(\Phi-\Psi)$ since photons traverse equal quantity of space and time. The galaxy-velocity cross-correlation $\beta \Upsilon_{\text {gg }}$ probed with RSD is sensitive to galaxy bias and the Newtonian potential $\Psi$. In GR and in absence of anisotropic stress, $\Phi=-\Psi$ so lensing is sensitive of $2 \nabla^{2} \Phi$. In the linear regime, the Poisson equation relates the potential to the matter density contrast $\delta$, such that $\nabla^{2} \Phi=-\nabla^{2} \Psi=\frac{3}{2} \Omega_{\mathrm{m} 0} H_{0}^{2} a^{-1} \delta$. This estimator therefore converges to $E_{\mathrm{G}}=\frac{\Omega_{\mathrm{m} 0}}{f}$ in the standard model.

In their seminal paper, Zhang et al. (2007) predicted deviations from GR with four alternative models: $\Lambda$ CDM, flat (Dvali et al. 2000, hereafter DGP), $f(R)$ gravity (Bean et al. 2007), and TeVeS/MOND (Bekenstein 2004). Apart from the TeVeS/MOND model, which introduces a wavelength difference between dynamical and lensing power-spectra, all other models add at most $10 \%$ deviations compared to GR predictions. Leonard et al. (2015) reached similar conclusions with other models based on the empirical extension of the Poisson equations with the commonly used parameters $\Sigma(a)$ and $\mu(a)$ (Amendola et al. 2008; Ferreira \& Skordis 2010). Most importantly, these authors found that details of the analysis (e.g. integration length along the line of sight for projected estimators) could mimic deviations similar to those predicted with alternative models of gravity, thus the need for a careful study of these biases. In any case with $20 \%-30 \%$ precision, current datasets are not yet at the level of accuracy required to observe these deviations, and unsurprisingly no deviation to GR predictions has been detected so far (Blake et al. 2016; Pullen et al. 2016; de la Torre et al. 2017; Alam et al. 2017; Amon et al. 2018).

Nowadays, cosmological analyses require measurements with exquisite control of systematic errors, at all levels from data acquisition to cosmological model inference. The wide range of expertise needed to reach the requirements is demonstrated by the size of the on-going and forthcoming cosmological experiments such as Dark Energy Survey (Dark Energy Survey Collaboration 2005), the Kilo Degree Survey (Hildebrandt et al. 2017), the Hyper-Suprime Cam survey (Aihara et al. 2018), the extended Baryonic Oscillation Sky Survey (Dawson et al. 2013), the Prime Focus Spectrograph project (Sugai et al. 2012), the Dark Energy Survey Instrument project (DESI Collaboration 2016a,b), the Large Scale Synoptic Telescope (LSST Dark Energy Science Collaboration 2012), and the Euclid mission Laureijs et al. (2011).

In this paper, we extend the Leauthaud et al. (2017) analysis (hereafter L17), by adding RSD measurements of CMASS galaxies from the Baryon acoustic Oscillation Spectroscopic Survey (BOSS) to GGL measurements in the CFHT-Stripe 82 and CFHTLS fields. Thanks to refined simulations, we precisely quantify systematic errors, and thus manage to reconcile real and simulated measurements of clustering and lensing. The work presented builds on the theoretical model and joint RSD and GGL analysis developed in de la Torre et al. (2017, hereafter DLT17).

The outline of the paper is as follows. First we present our galaxy bias model, and its inclusion in standard clustering and lensing estimators. Next, we present our datasets and measurement estimators. Our tests on simulations are presented in Sect. 5, and our estimates of the cosmological parameters in Sect. 6. Finally, we present our measurement of $E_{\mathrm{G}}$ and conclude. Systematic errors are discussed in the Appendix. Unless otherwise mentioned, we express the GGL projected densities $\Sigma_{\text {gm }}$ and distances in comoving coordinates. We assume the fiducial $\Lambda$ CDM-GR cosmology with flat universe, $\Omega_{\mathrm{m}}=0.31$, $h=0.6777, \Omega_{\mathrm{b}}=0.048, \sigma_{8}=0.82$ (Planck Collaboration XIII 2016).

\section{Method}

In the following, we compute the RSD two-point galaxy correlation functions in configuration space. We decomposed the three-dimensional galaxy separation vector $\mathbf{s}$ into polar $(s, \mu)$ or Cartesian $\left(r_{\mathrm{p}}, \pi\right)$ coordinates in the frame defined by the line of sight and the normal to it, where $s$ is the norm of $\mathbf{s}, \mu$ is the cosine of the angle between $\mathbf{s}$ and the line of sight, $\pi$ and $r_{\mathrm{p}}$ are the projections of $\mathbf{s}$ on the line of sight and its normal, respectively. In the flat-sky approximation, the transformation between Cartesian and polar coordinates is $\mu=\pi / s, r_{\mathrm{p}}=\sqrt{s^{2}-\pi^{2}}$ (Fisher et al. 1994). Conversely, the GGL formalism is defined in real space, where the separation vector $\mathbf{r}$ is decomposed into Cartesian coordinates $(R, \chi)$, where $\chi$ and $R$ are the projections of $\mathbf{r}$ on the line of sight and its normal, respectively. In GGL, the radial window function of integration is hundreds of $h^{-1} \mathrm{Mpc}$, and the effects of RSD can safely be neglected (Baldauf et al. 2010). Hereafter, we assume that $R$ in the model corresponds to $r_{\mathrm{p}}$ in the observations.

\subsection{Galaxy bias model}

In this work, we want to measure the growth rate $f$ and amplitude of the matter power spectrum $\sigma_{8}$ with GGL and galaxy-clustering measurements. These measurements are not typically estimated at the same scale. While the GGL signal typically emerges in the range of transverse distances $0.1<r_{\mathrm{p}}<20 h^{-1} \mathrm{Mpc}$, the galaxy clustering signal rises between $10<r_{\mathrm{p}}<100 h^{-1} \mathrm{Mpc}$. To maximize the overlap between these 
two observables in the non-linear regime, we adopted the fourth order perturbation model in the initial density field as proposed by McDonald \& Roy (2009). Assuming homogeneity and isotropy in the density field, they derived the following expression for the halo-matter power spectrum:

$$
\begin{aligned}
P_{\mathrm{gm}}(k)= & b_{1} P_{\delta \delta}(k)+b_{2} P_{b_{2}, \delta}(k) \\
& +b_{s^{2}} P_{b_{s^{2}}, \delta}(k)+b_{3 \mathrm{nl}} \sigma_{3}^{2}(k) P_{\mathrm{lin}}(k),
\end{aligned}
$$

where $P_{\delta \delta}$ and $P_{\text {lin }}$ represent the non-linear and linear matter power spectra respectively; $P_{b_{2}, \delta}$ and $P_{b_{s^{2}}, \delta}$ are the one-loop power spectra between the density field $\delta$, its derivative and the variance of the tidal tensor field $s(\mathbf{x})$. The term $b_{3 \mathrm{nl}} \sigma_{3}^{2}(k)$ includes various third order terms of the galaxy bias model (see McDonald \& Roy 2009, for more details). Assuming coevolution between the halo and matter density fields, and the bias being purely local in Lagrangian space at initial conditions, Baldauf et al. (2012) computed the second order halo density field in both Eulerian and Lagrangian space and found the relation $b_{s^{2}}=-4 / 7\left(b_{1}-1\right)$. Under the same assumptions as above to compute $b_{s^{2}}$, Saito et al. (2014) obtained the relation $b_{3 \mathrm{nl}}=$ $32 / 315\left(b_{1}-1\right)$. The analytical expressions for all these terms are given in Appendix A of DLT17.

\subsection{Galaxy-galaxy lensing model}

The measured GGL differential excess surface density is defined as

$\Delta \Sigma_{\mathrm{gm}}(R)=\bar{\Sigma}_{\mathrm{gm}}(R)-\Sigma_{\mathrm{gm}}(R)$,

where the mean projected surface density can be read as

$\bar{\Sigma}_{\mathrm{gm}}(R)=\frac{2}{R^{2}} \int_{0}^{R} \Sigma_{\mathrm{gm}}(r) r \mathrm{~d} r$,

and $\Sigma_{\mathrm{gm}}(R)$ is the projected surface density defined as a function of the galaxy-matter cross-correlation function (Dvornik et al. 2018)

$\Sigma_{\mathrm{gm}}(R)=\Omega_{\mathrm{m}} \rho_{\mathrm{c}} \int_{-\infty}^{\infty} \xi_{\mathrm{gm}}\left(\sqrt{R^{2}+\chi^{2}}\right) \mathrm{d} \chi$,

where the mean matter density $\rho_{\mathrm{m}}=\Omega_{\mathrm{m}} \rho_{\mathrm{c}}=3 \Omega_{\mathrm{m} 0} H_{0}^{2} / 8 \pi G$ is constant in comoving coordinates. The galaxy-matter crosscorrelation function $\xi_{\mathrm{gm}}$ is obtained from the Fourier transform of the galaxy-matter power spectrum $P_{\mathrm{gm}}(k)$ defined above.

In practice, we used an FFTLog unbiased Hankel transform with parameter $\mu=\frac{1}{2}$ in logarithmic space to perform the Fourier transform ${ }^{1}$. We truncated the power spectrum at $k_{\min }=10^{-5}$ and $k_{\max }=1000$ to minimize cut-off aliasing during the FFT operation, and we spline-interpolated the resulting correlation function to obtain the desired binning.

\subsection{Redshift space distortions model}

In this work, we used the Taruya et al. (2010) model to describe the RSD effect. In the ideal case in which galaxies are perfect tracers of the matter density field, this model takes the form

$$
\begin{aligned}
P^{s}(k, \mu)= & D\left(k \mu \sigma_{v}\right)\left[P_{\delta \delta}(k)+2 \mu^{2} f P_{\delta \theta}(k)+\mu^{4} f^{2} P_{\theta \theta}(k)\right. \\
& \left.+C_{A}(k, \mu, f)+C_{B}(k, \mu, f)\right],
\end{aligned}
$$

\footnotetext{
1 http://casa.colorado.edu/ajsh
}

where $\theta$ is the divergence of the velocity field defined as $\theta=$ $-\nabla \cdot \mathbf{v} /(a H f)$. The values $P_{\delta \delta}, P_{\theta \theta}$, and $P_{\delta \theta}$ are the non-linear matter density, velocity divergence, and density-velocity divergence power spectra, respectively; $C_{A}(k, \mu, f)$ and $C_{B}(k, \mu, f)$ terms derive from the general anisotropic power spectrum of matter and their expressions are given in Taruya et al. (2010) and de la Torre \& Guzzo (2012).

The damping function $D\left(k \mu \sigma_{v}\right)$ essentially (but not only) describes the Fingers of God effect on the two-point correlation function, and we modelled it as a Lorentzian damping in Fourier space, i.e.

$D\left(k, \mu, \sigma_{v}\right)=\left(1+k^{2} \mu^{2} \sigma_{v}^{2}\right)^{-1}$,

where $\sigma_{v}$ represents an effective pairwise velocity dispersion that we fitted for and then treated as a nuisance parameter.

This model can be generalized to the case of biased tracers, by including our bias model. Hence, we obtain (Beutler et al. 2014; Gil-Marín et al. 2014)

$$
\begin{aligned}
P_{\mathrm{g}}^{s}(k, \mu)= & D\left(k \mu \sigma_{v}\right)\left[P_{\mathrm{gg}}(k)+2 \mu^{2} f P_{\mathrm{g} \theta}+\mu^{4} f^{2} P_{\theta \theta}(k)\right. \\
& \left.+C_{A}\left(k, \mu, f, b_{1}\right)+C_{B}\left(k, \mu, f, b_{1}\right)\right],
\end{aligned}
$$

where,

$$
\begin{aligned}
P_{\mathrm{gg}}(k)= & b_{1}^{2} P_{\delta \delta}(k)+2 b_{2} b_{1} P_{b_{2}, \delta}(k)+2 b_{s^{2}} b_{1} P_{b_{s^{2}}, \delta}(k) \\
& +b_{2}^{2} P_{b_{2}, b_{2}}(k)+2 b_{2} b_{s^{2}} P_{b_{2}, b_{s^{2}}}(k)+b_{s^{2}}^{2} P_{b_{s^{2}}, b_{s^{2}}}(k) \\
& +2 b_{1} b_{3 \mathrm{nl}} \sigma_{3}^{2}(k) P_{\operatorname{lin}}(k) \\
P_{\mathrm{g} \theta}(k)= & b_{1} P_{\delta \theta}(k)+b_{2} P_{b_{2}, \theta}(k) \\
& +b_{s^{2}} P_{b_{s^{2}}, \theta}(k)+b_{3 \mathrm{nl}} \sigma_{3}^{2}(k) P_{\operatorname{lin}}(k) .
\end{aligned}
$$

In the above equations $P_{b_{2}, \delta}, P_{b_{s^{2}}, \delta}, P_{b_{2}, b_{2}}, P_{b_{2}, b_{s^{2}}}, P_{b_{s^{2}}, b_{s^{2}}}$ and $\sigma_{3}^{2}(k)$ are one-loop integrals, of which analytical expressions can be found in Appendix A of DLT17. We computed the linear matter power spectrum $P_{\text {lin }}$ using the class Bolzmann code (Lesgourgues 2011), and the non-linear matter power spectrum $P_{\delta \delta}$ using the semi-analytic prescriptions HALOFIT (Smith et al. 2003; Takahashi et al. 2012). To predict the velocity spectra $P_{\theta \theta}$ and $P_{\delta \theta}$, we use the nearly universal fitting functions from Bel et al. (2019), already used in DLT17 and Pezzotta et al. (2017). These are built such that they converge to $P_{\text {lin }}$ at large scales, but reproduce non-linearities at small scales. Pezzotta et al. (2017) highlighted that adding a redshift dependency with $\sigma_{8}(z)$ such that

$P_{\theta \theta}(k)=P_{\text {lin }}(k) \exp \left[-k p_{1} \sigma_{8}^{p_{2}}(z)\right]$,

and

$P_{\delta \theta}(k)=\sqrt{P_{\delta \delta} P_{\operatorname{lin}}(k) \exp \left[-k p_{3} \sigma_{8}^{p_{4}}(z)\right]}$,

was helping. The coefficients $\left(p_{1}=1.906, p_{2}=2.163, p_{3}=\right.$ $2.972, p_{4}=2034$ ) were deduced from a fit to measurements performed on the DEMNUni simulations (dark energy and massive neutrino universe). These two fitting functions are accurate within $5 \%$ to the measurements in simulations and appear to be insensitive to the presence of neutrinos (Carbone et al. 2016). The overall degree of non-linearity in these terms is therefore solely controlled by $\sigma_{8}(z)$, which is left free when fitting the model to observations. Although these fitting functions possibly duplicate a fraction of the high-order modes included in the perturbation theory model above, we demonstrate in DLT17 and in Sect. 5 below that it does not bias significantly our cosmological estimates given data uncertainties. 
Finally, we obtain the multipole moments of the anisotropic correlation functions in configuration space

$\xi_{\ell}^{s}(s)=i^{\ell} \int \frac{k^{2}}{2 \pi^{2}} P_{\ell}^{s}(k) j_{\ell}(k s) \mathrm{d} k$,

where $j_{\ell}(x)$ is the spherical Bessel function and $P_{\ell}^{s}(k)$ is the anisotropic power-spectrum multipole moment of order $\ell$ defined as

$P_{\ell}^{s}(k)=\frac{2 \ell+1}{2} \int_{-1}^{1} P_{\mathrm{g}}^{s}(k, \mu) L_{\ell}(\mu) \mathrm{d} \mu$,

where $L_{\ell}(x)$ are the Legendre polynomial of order $\ell$.

At linear scales, $f$ and $\sigma_{8}$ are degenerate, but extending to non-linear scales with the Taruya et al. (2010) model, $b_{1}^{2} f \sigma_{8}^{4}$, $b_{1} f^{2} \sigma_{8}^{4}$, and $f^{3} \sigma_{8}^{4}$ appear in the calculation of the correction terms $C_{A}$ and $C_{B}$, and hence help break the degeneracy. Accordingly, in our model $\left(f, b_{1}, b_{2}, \sigma_{v}, \sigma_{8}\right)$ are treated as separate parameters in the fit (de la Torre \& Guzzo 2012).

\subsection{Spectroscopic redshift uncertainties}

It is worth mentioning that redshift errors can potentially affect the anisotropic RSD signal. They have the same effect as galaxy random motions in virialized objects. We model the redshift errors by multiplying the anisotropic power spectrum by the Fourier transform of a Gaussian damping function of the form

$G\left(k, \mu, \sigma_{z}\right)=\exp \left(-\frac{k^{2} \mu^{2} \sigma_{z}^{2}}{2}\right)$,

such that our predicted signal can be finally written as

$\widehat{P_{\mathrm{g}}^{s}}=G\left(k, \mu, \sigma_{z}\right) P_{\mathrm{g}}^{s}$.

Bolton et al. (2012) measured the error on the estimated spectroscopic velocities, thanks to multiple observations of the same CMASS galaxies, and found approximately $\delta_{v}=$ $32 \mathrm{~km} \mathrm{~s}^{-1}$, which translates to $\sigma_{z}=0.797 h^{-1} \mathrm{Mpc}$ in comoving distances at redshift $z=0.57$ with our fiducial cosmology. This effect is therefore negligible, but we included it to have a cleaner estimate of $\sigma_{v}$.

\subsection{Suppressing small-scale modelling uncertainties}

Although considered as sufficient for galaxy-clustering analysis, we find that our weak-lensing (WL) model deviates from our measurements with simulated data at scales $r_{\mathrm{p}} \sim 3 h^{-1} \mathrm{Mpc}$ (see Fig. 7 in DLT17). In order to damp the contribution of any signal below a given cut-off radius $R_{0}$, we computed the annular differential excess surface density (ASAD) estimator from the data (Baldauf et al. 2010). For the lensing observable $\Delta \Sigma_{\mathrm{gm}}\left(r_{\mathrm{p}}\right)$, it is given by

$\Upsilon_{\mathrm{gm}}\left(r_{\mathrm{p}}, R_{0}\right)=\Delta \Sigma_{\mathrm{gm}}\left(r_{\mathrm{p}}\right)-\left(\frac{R_{0}}{r_{\mathrm{p}}}\right)^{2} \Delta \Sigma_{\mathrm{gm}}\left(R_{0}\right)$,

and for the galaxy clustering

$\Upsilon_{\mathrm{gg}}\left(r_{\mathrm{p}}, R_{0}\right)=\rho_{c}\left[\frac{2}{r_{\mathrm{p}}^{2}} \int_{R_{0}}^{r_{\mathrm{p}}} \mathrm{d} r r w_{\mathrm{p}}(r)-w_{\mathrm{p}}\left(r_{\mathrm{p}}\right)+\frac{R_{0}^{2}}{r_{\mathrm{p}}^{2}} w_{\mathrm{p}}\left(R_{0}\right)\right]$.

These two estimators become useful to estimate $E_{\mathrm{G}}$ in the following. We derive the projected correlation $w_{\mathrm{p}}\left(r_{\mathrm{p}}\right)$ from the projection of the multipole decomposition of the correlation function in redshift space $\xi_{\ell}^{s}(s)$

$$
\begin{aligned}
w_{\mathrm{p}}\left(r_{\mathrm{p}}\right)= & 2 \sum_{\ell=0}^{2} \alpha_{2 \ell} \int_{0}^{\pi_{\max }} \mathrm{d} \pi \xi_{2 \ell}^{s}\left(\sqrt{r_{\mathrm{p}}^{2}+\pi^{2}}\right) \\
& \times L_{2 \ell}\left(\frac{\pi}{\sqrt{r_{\mathrm{p}}^{2}+\pi^{2}}}\right) .
\end{aligned}
$$

The $\alpha_{2 \ell}$ coefficients are given in Baldauf et al. (2010)

$$
\begin{aligned}
& \alpha_{0}(\beta)=1+\frac{2}{3} \beta+\frac{1}{5} \beta^{2}, \\
& \alpha_{2}(\beta)=\frac{4}{3} \beta+\frac{4}{7} \beta^{2}, \\
& \alpha_{4}(\beta)=\frac{8}{35} \beta^{2} .
\end{aligned}
$$

We integrate along the line of sight up to $\pi_{\max }=40 h^{-1} \mathrm{Mpc}$ to match the integration length used with the data (see the estimators Sect. 4.2). According to Singh et al. (2019), they found consistent results whether they use $\pi_{\max }=50 h^{-1} \mathrm{Mpc}$ or $100 h^{-1} \mathrm{Mpc}$. Given the low number CMASS galaxies in this analysis, we set $\pi_{\max }=40 h^{-1} \mathrm{Mpc}$ to minimize the noise.

The ASAD can also be predicted from theory. For the lensing part, $\Upsilon_{\mathrm{gm}}\left(r_{\mathrm{p}}, R_{0}\right)$ is obtained by filtering the cross-correlation function $\xi_{\mathrm{gm}}(r)$

$\Upsilon_{\mathrm{gm}}\left(r_{\mathrm{p}}, R_{0}\right)=\int_{0}^{\infty} \xi_{\mathrm{gm}}(r) W_{\Upsilon}\left(r, r_{\mathrm{p}}, R_{0}\right) \mathrm{d} r$,

with the window function $W_{\Upsilon}\left(x, r_{\mathrm{p}}, R_{0}\right)$ (Baldauf et al. 2010) defined as

$$
\begin{aligned}
W_{\Upsilon}\left(x, r_{\mathrm{p}}, R_{0}\right)= & \frac{4 x}{r_{\mathrm{p}}^{2}}\left(\sqrt{x^{2}-R_{0}^{2}} \Theta\left(x-R_{0}\right)-\sqrt{x^{2}-r_{\mathrm{p}}^{2}} \Theta\left(x-r_{\mathrm{p}}\right)\right) \\
& -\frac{2 x}{r_{\mathrm{p}}^{2}}\left(\frac{r_{\mathrm{p}}^{2} \Theta\left(x-r_{\mathrm{p}}\right)}{\sqrt{x^{2}-r_{\mathrm{p}}^{2}}}-\frac{R_{0}^{2} \Theta\left(x-R_{0}\right)}{\sqrt{x^{2}-R_{0}^{2}}}\right)
\end{aligned}
$$

where $\Theta(x)$ is the Heaviside step function. In a similar manner, we computed $\Upsilon_{\mathrm{gg}}\left(r_{\mathrm{p}}, R_{0}\right)$ by simply replacing $\xi_{\mathrm{gm}}(r)$ by $\xi_{\mathrm{gg}}(r)$ in Eq. (23). We included the RSD effect in the calculation of $\Upsilon_{\mathrm{gg}}\left(r_{\mathrm{p}}\right)$. In both cases, we integrated in logarithmic scale up to $r_{\text {max }}=100 h^{-1} \mathrm{Mpc}$.

We note that we do not include intrinsic alignment in our modelling. This choice is motivated by the marginal constraints obtained in Joudaki et al. (2017) on the amplitude of this effect $A_{\text {IA }}=1.67_{-0.49}^{+0.50}$, with small-scale cut on $\gamma_{\mathrm{t}}$ at $\theta>12$ arcmin. Since we applied the small-scale $\Upsilon$ filter, we anticipate very little constraint on this parameter as well, at a significant additional computing cost.

\subsection{Alcock-Paczynski effect}

We may mention that additional distortions can occur in the correlation functions owing to possible differences between the true and the fiducial cosmological models used to compute the distances. This effect was first identified by Alcock \& Paczynski (1979; hereafter AP) as a means to constrain the cosmological model. However these distortions degenerate with the RSD effect and considerably limit the constraining power of the AP effect (Ballinger et al. 1996; Matsubara \& Suto 1996). 
Fortunately, the scale-dependence of the AP and RSD effects differ and thus allow us this degeneracy to break (Seo \& Eisenstein 2003; Blake et al. 2011; Chuang \& Wang 2012).

In this work, we adopted the AP model proposed by Xu et al. (2013). The isotropic and anisotropic distortions are expressed respectively as

$$
\begin{aligned}
& \alpha=\left(\frac{D_{A}^{2}}{D_{A}^{\prime 2}} \frac{H^{\prime}}{H}\right)^{1 / 3}, \\
& 1+\epsilon=\left(\frac{D_{A}^{\prime}}{D_{A}} \frac{H^{\prime}}{H}\right)^{1 / 3},
\end{aligned}
$$

where quantities computed with the fiducial cosmology as denoted with primes. Those parameters modify the transverse and the radial distances such that

$\pi^{\prime}=\alpha(1+\epsilon)^{2} \pi$

$r_{\mathrm{p}}^{\prime}=\alpha(1+\epsilon)^{-1} r_{\mathrm{p}}$

Given these distortions, the observed redshift-space monopole and quadrupole expressed in configuration space become

$$
\begin{aligned}
\xi_{0}^{\prime}\left(s^{\prime}\right)= & \xi_{0}(\alpha s)+\frac{2}{5} \epsilon\left[3 \xi_{2}(\alpha s)+\frac{\mathrm{d} \xi_{2}(\alpha s)}{\mathrm{d} \ln (s)}\right] \\
\xi_{2}^{\prime}\left(s^{\prime}\right)= & 2 \epsilon \frac{\mathrm{d} \xi_{0}(\alpha s)}{\mathrm{d} \ln (s)}+\left(1+\frac{6}{7} \epsilon\right) \xi_{2}(\alpha s)+\frac{4}{7} \epsilon \frac{\mathrm{d} \ln (\alpha s)}{\mathrm{d} \ln (s)} \\
& +\frac{4}{7} \epsilon\left[5 \xi_{4}(\alpha s)+\frac{\mathrm{d} \xi_{4}(\alpha s)}{\mathrm{d} \ln (s)}\right],
\end{aligned}
$$

The GGL estimator becomes

$\Upsilon_{\mathrm{gm}}^{\prime}\left(R^{\prime}\right)=\Upsilon_{\mathrm{gm}}\left[\alpha(1+\epsilon)^{-1} R\right]$

\section{Data}

In our GGL analysis, the lenses are the CMASS galaxies and the sources are galaxies in the CFHTLens and CFHT-Stripe 82 WL catalogues. Lenses have spectroscopic redshifts and sources have photometric redshifts. For each lens, we can then discard all uncorrelated foreground sources and use the background sources to estimate the lensing signal. The final GGL measurement is the average of the signals for each lens.

\subsection{Weak-lensing datasets}

\subsubsection{CFHTLens catalogue}

In 2013, the CFHTLenS team released a public WL catalogue covering an area of $154 \mathrm{sq}$. deg in four wide fields (W1, W2, W3, and W4; Erben et al. 2013; Heymans et al. 2012). So far, the depth of the input CFHT Legacy Survey imaging is unrivaled, with a $5 \sigma$ point source limiting magnitude $i_{\mathrm{AB}} \sim 25.5$. The LENSFIT algorithm is used to measure the shape of every object detected with $i_{\mathrm{AB}}<24.7$. Then, we selected galaxies with good shape measurements (FITCLASS $=0$ and WEIGHT $>3$ ).

We obtained photometric redshifts from five optical band photometry $u, g, r, i, z$ and reach a precision of about $5 \%$ up to $z \sim 1$ (Hildebrandt et al. 2012). Galaxy-galaxy lensing measurements can be significantly biased by inaccurate photometric redshifts (Nakajima et al. 2012). We computed the photometric redshift bias estimator $\left\langle b_{z}\right\rangle$, based on spectroscopic and photometric catalogues matched in position, and averaged over the CMASS redshift distribution (see appendix details). Since the spectroscopic calibration sample is significantly shallower than the photometric sample, we discarded galaxies fainter than the 90\% completeness limit of the spectroscopic sample (see below), i.e. we only kept galaxies brighter than $i_{\mathrm{AB}}<24$. After this selection, we obtained $\left\langle b_{z}\right\rangle=+0.003 \pm 0.003,\left\langle b_{z}\right\rangle=-0.014 \pm 0.004$ and $\left\langle b_{z}\right\rangle=+0.022 \pm 0.003$ in fields $\mathrm{W} 1, \mathrm{~W} 3$, and $\mathrm{W} 4$, respectively. We discarded field W2 because it only contains 200 CMASS galaxies on its northern edge.

Our final catalogue contains 3.5 millions galaxies over an effective area of about $127 \mathrm{sq}$. deg. The galaxy density ${ }^{2}$ is $n_{\text {eff }}=$ 7.0 galaxies $\operatorname{arcmin}^{-2}$. The median redshift is $z_{\text {med }}=0.70$.

\subsubsection{CFHT-Stripe 82 catalogue}

The CFHT-Stripe 82 survey (CS82; Moraes et al. 2014) is an $i$-band imaging survey containing 173 tiles (PIs: J.-P. Kneib, A. Leauthaud, M. Makler, L. Van Waerbeke). It covers about 160 sq. deg of the Sloan Digital Sky Survey (SDSS, Gunn et al. 2006) stripe 82 region, with a $5 \sigma$ point-source magnitude limit $i_{\mathrm{AB}} \sim 24.1$, and a mean seeing of $0.6^{\prime \prime}$. The effective area is 129.2 sq. deg after masking out bright stars and other image artefacts (L17). We used a new version 3.0 of the shape catalogue that has shapes measured with LENSFIT down to magnitude $i_{\mathrm{AB}}<24.7$. This new version benefits from internal calibration in LENSFIT based on image simulations inherited from the CFHTLenS project. Shape measurements are accurate at the $2 \%$ level, without relying on any additional linear correction. In addition, this new catalogue contains about $40 \%$ more galaxies, mostly because of a better handling of galaxy de-blending and instrument artefacts in LENSFIT (L. van Waerbeke, priv. comm.).

Photometric redshifts in the original version of the catalogue (Bundy et al. 2015) were computed with BPZ (Benítez 2000) using ugriz from the Stripe 82 co-adds (Annis et al. 2014) and UJHK from UKIDSS. We use nearest-neighbour interpolation in sky coordinates, $i$ magnitude, and $g-r, r-i, i-z$ colour space to get photometric redshifts for the new galaxies. We verified that the redshift distribution is unchanged. We applied the same procedure as in the CFHTLS fields to estimate the bias due to photometric redshifts in our GGL measurements. However, given the relatively shallow spectroscopic survey coverage of the CS82 field compared to CFHTLS fields (90\% completeness reached at $i_{\mathrm{AB}}=22.5$ ), we are forced to select galaxies only down to $i_{\mathrm{AB}}<22.5$. Although this cut is quite severe, it allow us to confidently model and correct photometric redshift bias in this field. The lack of deeper spectroscopic information prevents us from exploiting the complete WL catalogue. For $i_{\mathrm{AB}}<22.5$ sources and CMASS lenses, we find a bias $b_{z}=-0.028 \pm 0.006$. In contrast to L17, we apply no cut based on the oDD quality flag because we find it has no impact on our lensing measurements given our stringent cut in magnitude. Our final catalogue contains 2.2 million galaxies. The galaxy density is $n_{\mathrm{eff}}=4.7$ galaxies $\operatorname{arcmin}^{-2}$. The median redshift is $z_{\text {median }}=0.53$.

\subsection{Spectroscopic dataset: BOSS CMASS sample}

The BOSS spectroscopic survey (Eisenstein et al. 2011) is a programme of the SDSS project. The constant (stellar) mass

\footnotetext{
2 We use the definition $n_{\mathrm{eff}}=\frac{1}{\Omega} \frac{\left(\sum w_{i}\right)^{2}}{\sum w_{i}^{2}}$ (Heymans et al. 2012), where $w_{i}$
} is a galaxy weight and $\Omega$ is the opening angle. 


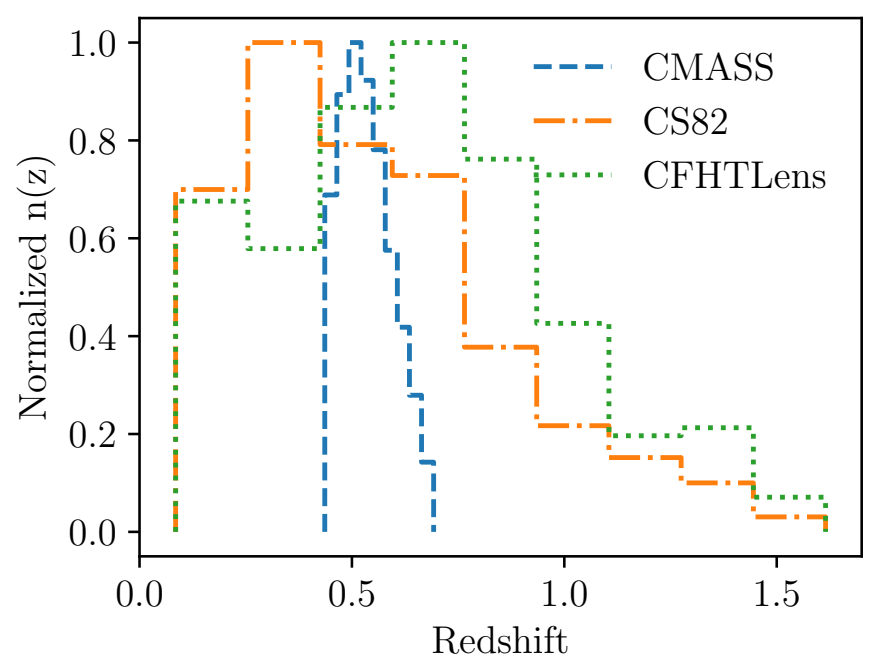

Fig. 1. Redshift distribution of CMASS galaxies (blue) compared to CS82 (orange) and CFHTLens (green) source distributions, after WL selection has been performed. WL $n(z)$ are based on photometric redshifts (see text for details).

(CMASS) galaxy sample is one of the galaxy samples observed in this survey. This sample consists of galaxies selected with SDSS photometry, such that they lie in the redshift range $0.43<z<0.7$ and represent a sample of galaxies approximately volume-limited in stellar mass (Reid et al. 2016). Early clustering analysis found that CMASS galaxies lie in massive haloes and have a mean halo mass of $2.6 \times 10^{13} h^{-1} M_{\odot}$, a large-scale bias of $b \sim 2.0$, and a satellite fraction of $10 \%$ (White et al. 2011).

We used the public DR12v5 version of the CMASS catalogue (Alam et al. 2015). The galaxy surface density is about $100 \mathrm{deg}^{-2}$ (Reid et al. 2016). We only considered CMASS overlapping with our four lensing fields, i.e. covering an area 250 sq. deg. Our catalogue of lenses contains 28039 CMASS galaxies, distributed as reported in Table 1. The redshift distribution of CMASS galaxies compared to CS82 and CFHTLens lensing sources is shown in Fig. 1.

In spite of a careful photometric selection, the observed CMASS galaxy sample remains contaminated by various observational effects (Ross et al. 2012). We took these into account by applying the galaxy weights $w_{\mathrm{g}}=w_{\text {star }} w_{\text {see }}\left(w_{z f}+w_{c p}-\right.$ 1) as defined in Ross et al. (2017). We also included the Feldman et al. (1994, hereafter FKP) weights with the parameter $P_{0}=20000 \mathrm{~h}^{-3} \mathrm{Mpc}^{3}$ (Ross et al. 2012), such that the noise in the power spectrum is minimum at the baryon acoustic oscillation scale $k=0.1 \mathrm{~h} \mathrm{Mpc}^{-1}$. Although not optimal for our study focussed on small-scale clustering, this value of $P_{0}$ allows for a consistent comparison with previous measurements. For consistency, we take the same value of $P_{0}$ for our mock catalogues and data. Finally, we used the DR12v5_random 0 catalogues trimmed to the regions overlapping with WL data.

\section{Measurement estimators}

\subsection{Galaxy-galaxy lensing estimation}

We computed $\Delta \Sigma_{\mathrm{gm}}\left(r_{\mathrm{p}}\right)$ using the estimator

$$
\begin{aligned}
\Delta \Sigma_{\mathrm{gm}}\left(r_{\mathrm{p}}\right)= & \frac{\sum_{\mathrm{l}, \mathrm{s}}^{N_{\mathrm{s}}} \Sigma_{\mathrm{cr}}\left(z_{\mathrm{l}}, z_{\mathrm{s}}\right) w_{\mathrm{l}, \mathrm{s}} \epsilon_{+}\left(r_{\mathrm{p}}\right)}{\sum_{\mathrm{l}, \mathrm{s}}^{N_{\mathrm{s}}} w_{\mathrm{l}, \mathrm{s}}} \\
& -\frac{\sum_{\mathrm{r}, \mathrm{s}}^{N_{\mathrm{r}, \mathrm{s}}} \sum_{\mathrm{cr}}\left(z_{\mathrm{r}}, z_{\mathrm{s}}\right) w_{\mathrm{r}, \mathrm{s}} \epsilon_{+}\left(r_{\mathrm{p}}\right)}{\sum_{\mathrm{r}, \mathrm{s}}^{N_{\mathrm{s}}} w_{\mathrm{r}, \mathrm{s}}},
\end{aligned}
$$

where $r_{\mathrm{p}}$ is the comoving transverse distance between the lens and the source at redshifts $z_{1}$ and $z_{\mathrm{s}}$, respectively. The subscript " $r$ " denotes the random catalogue of lensing objects. Our number of random objects $N_{\mathrm{r}}$ is ten times the number of lenses $N_{1}$. Their redshift distribution $n(z)$ is that from CMASS galaxies (Nuza et al. 2013). The subtraction of the random signal decreases the variance at large scales (Singh et al. 2017; Shirasaki et al. 2017). The value $\epsilon_{+}$represents the tangential component of a source ellipticity around a lens. The weight $w_{\mathrm{l}, \mathrm{s}}=\Sigma_{\mathrm{cr}}^{-2}\left(z_{\mathrm{l}}, z_{\mathrm{s}}\right) w_{\mathrm{s}}$ is the product of the shape measurement weight $w_{\mathrm{s}}$ from LENSFIT and the critical density. This inverse variance scheme downweights pairs which are close in redshift (Mandelbaum et al. 2006). The critical lensing density $\Sigma_{\mathrm{cr}}\left(z_{1}, z_{\mathrm{s}}\right)$ in comoving units is defined as

$\Sigma_{\mathrm{cr}}\left(z_{\mathrm{l}}, z_{\mathrm{s}}\right)=\frac{c^{2}}{4 \pi G\left(1+z_{\mathrm{s}}\right)^{2}} \frac{D_{\mathrm{S}}}{D_{\mathrm{LS}} D_{\mathrm{L}}}$,

where $D_{\mathrm{S}}, D_{\mathrm{LS}}, D_{\mathrm{L}}$ are the observer-source, lens-source, and observer-lens angular diameter distances ${ }^{3}$ We used the bestfit estimate of the photometric redshift to compute the distances, instead of the full probability distribution, as suggested in Blake et al. (2016). However, our approach described below and based on full ray-tracing simulations consistently takes this simplification into account.

\subsection{Anisotropic galaxy clustering estimation}

We computed the two-point galaxy correlation function in the polar and Cartesian coordinate systems. The anisotropy in the signal is due to the RSD effect we are after. The estimator is the same in each coordinate system and is defined as

$\xi(x, y)=\frac{\mathrm{GG}(x, y)-2 \mathrm{GR}(x, y)+\mathrm{RR}(x, y)}{\operatorname{RR}(x, y)}$,

where $(x, y)=(s, \mu)$ or $\left(r_{\mathrm{p}}, \pi\right)$. GG, GR and RR are the normalized number of pairs between galaxy-galaxy, galaxy-random, and random-random, respectively, at a given separation.

We compressed the information contained in $\xi(s, \mu)$ by projecting it on the Legendre polynomials using the expressions for the correlation-function multipole moments

$\xi_{\ell}(s)=\frac{2 \ell+1}{2} \int_{-1}^{1} \xi(s, \mu) L_{\ell}(\mu) \mathrm{d} \mu$,

where $L_{\ell}$ is the Legendre polynomial of order $\ell$. We used the monopole and quadrupole $\ell=(0,2)$ only because the higher order non-null multipoles are too noisy.

We also computed the projected correlation function $w_{\mathrm{p}}\left(r_{\mathrm{p}}\right)$ by projecting $\xi\left(r_{\mathrm{p}}, \pi\right)$ along the line of sight such that

$w_{\mathrm{p}}\left(r_{\mathrm{p}}\right)=2 \int_{0}^{\pi_{\max }} \xi\left(r_{\mathrm{p}}, \pi\right) \mathrm{d} \pi$

where we find the optimal value $\pi_{\max }=40 h^{-1} \mathrm{Mpc}$ to minimize the noise due to the limited number of pairs in our fields.

\subsection{Joint lensing and clustering likelihood}

We performed a maximum-likelihood analysis to derive the cosmological parameters from the GGL and RSD measurements. In

3 The factor $\left(1+z_{s}\right)^{2}$ is missed in Eq. (10) of de la Torre et al. (2017), but was properly taken into account in the calculations. 
Table 1. Number of CMASS galaxies per field, effective lensing area after masking and number of WL sources.

\begin{tabular}{cccccc}
\hline \hline Field & \# CMASS & $\begin{array}{c}\text { SDSS area } \\
\left(\mathrm{deg}^{2}\right)\end{array}$ & $\begin{array}{c}\text { SDSS field size } \\
(\mathrm{deg} \times \mathrm{deg})\end{array}$ & $\begin{array}{c}\text { Eff. area } \\
\left(\mathrm{deg}^{2}\right)\end{array}$ & $\begin{array}{c}\text { \# sources } \\
\left(\times 10^{6}\right)\end{array}$ \\
\hline S82 & 18675 & 219.8 & $87.6 \times 2.51$ & 129.2 & 2.19 \\
W1 & 3924 & 54.14 & $8.66 \times 6.3$ & 63.8 & 1.66 \\
W3 & 3694 & 41.91 & $11.5 \times 6.6$ & 44.2 & 1.26 \\
W4 & 1746 & 22.16 & $5.7 \times 5.61$ & 23.3 & 0.62 \\
\hline
\end{tabular}

each field $i$, we measured the data vector $\mathbf{d}^{i}=\left(\xi_{0}^{i}, \xi_{2}^{i}, \Upsilon_{\mathrm{gm}}^{i}\right)$ and we computed the likelihood function per field $\mathcal{L}^{i}$ such that

$-2 \ln \mathcal{L}^{i}=\left(\mathbf{d}^{i}-\mathbf{m}\right)^{T} \hat{\boldsymbol{\Phi}}^{i}\left(\mathbf{d}^{i}-\mathbf{m}\right)$,

where $\mathbf{m}$ is the model prediction, and $\hat{\boldsymbol{\Phi}}^{i}$ is the precision matrix estimated from the simulations.

Our four fields are statistically uncorrelated, and therefore the global likelihood is just the product of the individual likelihoods for each field, i.e.

$\mathcal{L}_{\text {tot }}=\mathcal{L}_{\mathrm{CS} 82} \times \mathcal{L}_{\mathrm{W} 1} \times \mathcal{L}_{\mathrm{W} 3} \times \mathcal{L}_{\mathrm{W} 4}$,

Field W4 partly overlaps with field S82, but this overlapping represents $<6 \%$ of the total area. In addition, CFHTLens catalogue used for W4 goes deeper than CS82 catalogue used for S82, thus decreasing further the correlation between the two fields.

\section{Simulations}

\subsection{Light cones and lensing mock catalogues}

In order to accurately estimate large-scale variance and possibly unveil new systematic errors, we produce light cones with the same geometry as the observed fields. We used the BigMultidark N-body simulation, as it appears to be a good compromise between particle resolution and cosmological volume $\left(m_{\mathrm{p}}=2.5 \times 10^{10} \mathrm{~h}^{-1} M_{\odot}, L_{\mathrm{box}}=2.5 \mathrm{~h}^{-1} \mathrm{Gpc}\right.$, Planck cosmology with $h=0.6777$; Klypin et al. 2016). Following the approach described in Giocoli et al. (2016), we simulated four fields, CS82, W1, W3, and W4, which have light cones extending up to redshift $z=2.3$ for the CFHT-LS fields and $z=2$ for CS82. We computed lensing properties, such as deflected positions, shear, and convergence, by ray tracing through 25 lens planes separated by $161 h^{-1}$ Mpc comoving (Giocoli et al. 2016) using the GLAMER ${ }^{4}$ code. The spatial resolution of the lensing maps is 6 arcsec.

\subsubsection{Lensing properties}

We simulated lensing catalogues of sources including survey mask, intrinsic shape, and photometric redshift noises. For survey mask, we placed the source galaxies are the location of observed sources. Thus, we naturally reproduced the footprint and the holes around bright stars and other artefacts of the real WL catalogue. Effects due to the intrinsic clustering of sources in projection were also included. We got the shear properties for each source by spatially interpolating the values from the shear maps computed with GLAMER. For sources intrinsic ellipticities, we randomly drew observed ellipticites $\epsilon^{\text {obs }}$ from the WL

\footnotetext{
4 Gravitational Lensing with Adaptive Mesh Refinement (Metcalf \& Petkova 2014).
}

catalogue, that we multiply by a random orientation $\phi^{\text {int }}$, such that $\epsilon_{1}^{\mathrm{int}}=\epsilon^{\mathrm{obs}} \cos \left(2 \phi^{\mathrm{int}}\right)$ and $\epsilon_{2}^{\mathrm{int}}=\epsilon^{\mathrm{obs}} \sin \left(2 \phi^{\mathrm{int}}\right)$.

\subsubsection{Photometric redshifts}

To simulate photometric redshifts with catastrophic failures, we designed a method related to that described in Lima et al. (2008), also referred as the direct calibration method (DIR) in the Kilo Degree Survey (KIDS, Hildebrandt et al. 2017). We start by estimating the true redshift distribution $n_{\text {true }}(z)$ for our CFHTLens $\left(i_{\mathrm{AB}}<24\right)$ and CS82 $\left(i_{\mathrm{AB}}<22.5\right) \mathrm{WL}$ catalogues from our spectroscopic calibration sample described in Appendix A. In practice, we computed the histograms of the WL and spectroscopic (ZP) catalogues in the magnitude-color space $(i, g-r, r-i, i-z)$, that we limited to the region $([18 ; 25],[-1 ; 3],[-1 ; 3],[-1 ; 3])$. We applied the same binning for both catalogues. For each bin of coordinates $\mathbf{m}$, we derive the weights $W(\mathbf{m})=N_{\mathrm{WL}}(\mathbf{m}) / N_{\mathrm{ZS}}(\mathbf{m})$, where $N$ is the number of sources per bin. We assume all sources in a bin have the same weight. Finally, we obtain the true distribution in redshift bin $i$ with the following sum:

$n_{\text {true }}\left(z_{i}\right)=\int \mathrm{d} \mathbf{m} N_{\mathrm{ZS}}\left(z_{i} \mid \mathbf{m}\right) W(\mathbf{m})$.

A drawback of this approach is that if spectroscopic selection does not cover part of the redshift range, then it truncates $n_{\text {true }}(z)$. However, we see in the following that the coverage is sufficient for our purpose.

Then, we compute the joint probability $P\left(z_{\mathrm{BPZ}}, z_{\mathrm{spec}}\right)$ for each field, as shown in Fig. 2. We observe that the spectroscopic redshift completeness at $z<0.5$ in field $\mathrm{W} 1$ and $\mathrm{W} 4$ is very low because most of the redshifts come from the CMASS sample. Fortunately, this has little impact on our simulation of photometric redshift noise because our analysis focusses on the crosscorrelation of CMASS galaxies with lensing sources at $z>0.5$. We also observe that the scatter in the $z_{\mathrm{BPZ}}$ of CS82 field is almost twice as large as in field W3, and differs between the three CFHTLens fields. This justifies our field-by-field treatment of the photometric redshift noise. Finally, we assign photometric redshifts to the simulated sources by randomly drawing a photometric redshift from $P\left(z_{\mathrm{BPZ}}, z_{\mathrm{spec}}\right)$, where we assume the spectroscopic redshift $z_{\text {spec }}$ is the true redshift that was assigned at the beginning of the procedure.

\subsection{Spectroscopic CMASS mock catalogues}

We adopted the sub-halo abundance matching (SHAM) procedure described in Rodríguez-Torres et al. (2016) to produce CMASS mock catalogues. Starting from the Rockstar public catalogues (Behroozi et al. 2013) ${ }^{5}$, we computed a scattered peak velocity $V_{\text {peak }}^{\text {scat }}=\left(1+\mathcal{N}\left(0, \sigma_{\text {SHAM }}\right)\right) V_{\text {peak }}$, where $\mathcal{N}$ is the

\footnotetext{
5 https://www.cosmosim.org/cms/simulations/bigmdpl/
} 

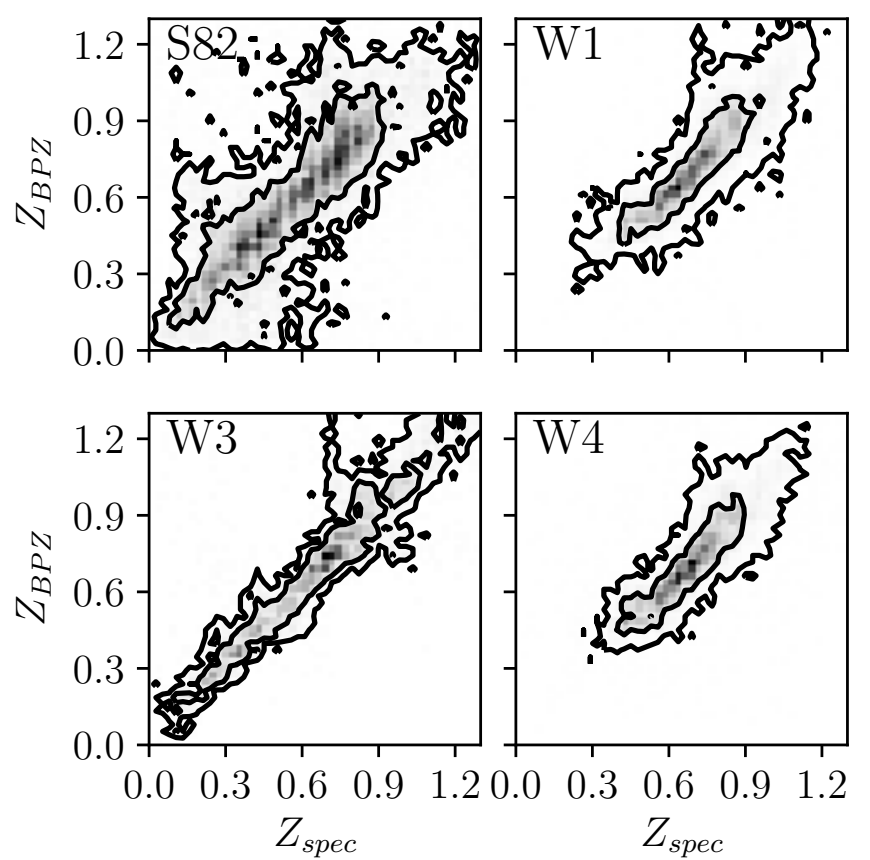

Fig. 2. Probability distribution of having a photometric redshift with BPZ and a spectroscopic redshift for each field. Contours are given for 1,2 , and $3 \sigma$ C.L.

normal distribution, and $\sigma_{\text {SHAM }}=0.31$. We also simulated the CMASS incompleteness in stellar mass and redshift, based on the stellar mass function (SMF) from the Portsmouth SED-FIT DR12 stellar mass catalogue with Kroupa initial mass function (Maraston et al. 2013). We binned the catalogue in 12 redshift intervals between $0.43<z<0.7$ and in 18 stellar mass bins between $10.5<\log _{10}\left(M * / M_{\odot}\right)<12.3$. Thus, we obtained a tabulated SMF that we can interpolate in stellar mass and redshift. Finally from cumulative stellar mass and halo mass functions, we constructed a number density matching such that $n_{\text {gal }}\left(>M_{*}^{i}\right)=n_{\text {halo }}\left(>V_{\text {peak }, i}^{\text {scat }}\right)$. Since different cosmologies were assumed in the Portsmouth catalogue and in the BigMultidark simulations, $h=0.73$ and $h=0.6777$ respectively, we renormalized the stellar masses to the BigMultidark cosmology. As shown in Fig. 3, our number densities for each of the four fields are in good agreement with the measurements from Anderson et al. (2012).

We also include the effect of peculiar velocities by summing together in redshift-space the halo position $\mathbf{r}_{\mathrm{c}}$ and the peculiar velocity vector $\mathbf{v}$ in real space using the relation $\mathbf{s}=\mathbf{r}_{\mathrm{c}}+\frac{\mathbf{v} \cdot \hat{\mathbf{r}}}{a H\left(z_{\text {real }}\right)}$, where $\hat{\mathbf{r}}$ is the line-of-sight unit vector, $a$ is the scale factor, and $H\left(z_{\text {real }}\right)$ is the Hubble parameter at redshift $z_{\text {real }}$, the redshift corresponding to $\mathbf{r}_{\mathrm{c}}$. Finally, we masked the borders of the square simulated fields W3 and W4 to reproduce their complex geometry, and we computed the FKP weights, assuming the same $P_{0}=20000 h^{-3} \mathrm{Mpc}^{3}$ as in the data. Since data have been corrected for fiber collision, redshift failure, stellar density, and seeing, we did not simulate these effects.

\subsection{Bias due to photometric redshift noise}

We computed successively the lensing signal for catalogues with and without photometric redshift noise, and compare the measurements in Fig. 4. We find that the large photometric scatter observed in field S82 (Fig. 2) seems to result in a bias of about
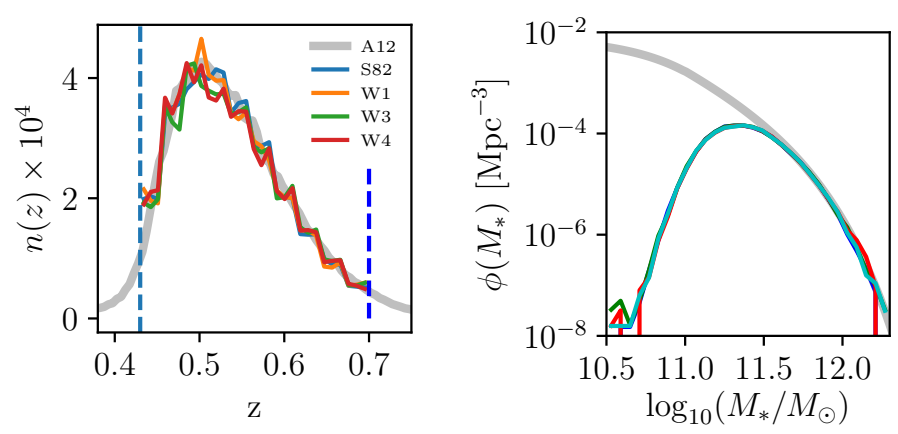

Fig. 3. Left panel: number density of CMASS mock galaxies for our 4 simulated fields. Limits of our analysis are indicated with blue dashed lines. Measurements from Anderson et al. (2012, A12) are in grey. Right panel: CMASS CMF for the 4 fields reproducing the observed incompleteness. The mock catalogue is complete at high mass in agreement with the model proposed in Rodríguez-Torres et al. (2016) in grey.

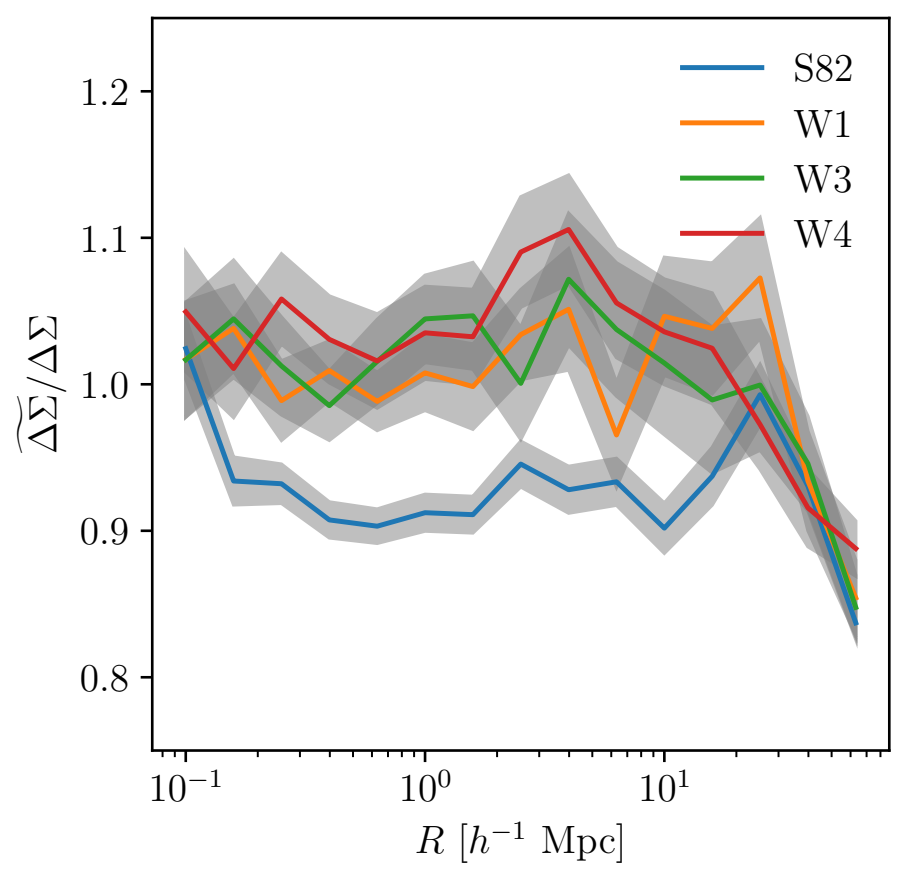

Fig. 4. Comparison of lensing measurements performed on simulated catalogues affected and not affected by photometric redshifts noise $\widetilde{\Delta \Sigma}$ and $\Delta \Sigma$, respectively. The grey shaded areas correspond to the uncertainties on the mean value obtained by resampling the multiple noises in different light cones.

$10 \%$ in the lensing signal at scales $R<10 h^{-1} \mathrm{Mpc}$, whereas the CFHTLens fields seem insignificantly affected. We argue that this might explain the discrepancy highlighted in L17 between lensing measurements obtained with real and mock data. Indeed, in the following, we show that our lensing measurements with mock data contaminated by photometric redshift noise are in agreement with real data.

\subsection{Bias from small-scale modelling}

We used the simulation to quantify the bias in the estimation of the cosmological parameters $f$ and $\Omega_{\mathrm{m}}$ due to our model prediction of the small scales. Successively, we cut data points of $\xi_{0}$ and $\xi_{2}$ at scales $s_{\min }=11.2,14.1$, and $17.8 h^{-1} \mathrm{Mpc}$, and $\Upsilon_{\mathrm{gm}}$ at scales $R_{0}=1.0$ and $1.5 h^{-1} \mathrm{Mpc}$. Overall, we find that the values $s_{\min }=17.8 h^{-1} \mathrm{Mpc}$ and $R_{0}=1 h^{-1} \mathrm{Mpc}$ provide the best 


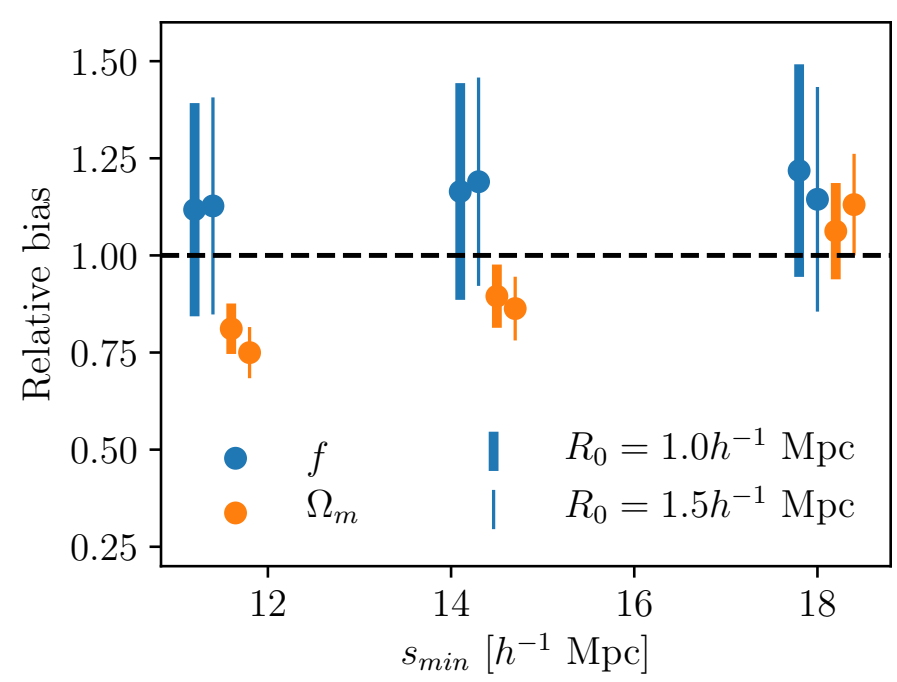

Fig. 5. Bias between recovered parameters $f$ and $\Omega_{\mathrm{m}}$ relative to the values used in the mocks as functions of the minimum scale of the multipoles $s_{\min }$, and the cut-off radius of the $\Upsilon\left(R, R_{0}\right)$ lensing estimator. Values of $s_{\min }=17.8 h^{-1} \mathrm{Mpc}$ and $R_{0}=1.0 h^{-1} \mathrm{Mpc}$ provide unbiased estimates of $f$ and $\Omega_{\mathrm{m}}$. All measurements were performed without tapering smoothing in the covariance matrices.

compromise between systematic bias and statistical precision as can be seen in Fig. 5.

\subsection{Covariance matrices}

To obtain an unbiased estimate of the precision matrices, we need minimal errors in the covariance matrices and therefore a large number of mock catalogues. Noise in the covariance matrices increases the errors on the model parameter estimation (see e.g. Taylor \& Joachimi 2014). Unfortunately, we were limited by the size of our simulation box $L=2 h^{-1} \mathrm{Gpc}$. Escoffier et al. (2016) proposed a method to increase the number of mocks, based on Jackknife resampling of the mock catalogues (see Table 2). Following their prescription, we split each catalogue into $N_{\mathrm{JK}}$ spatial subregions and measured the clustering and lensing signals in each Jackknife subsample using estimators given in Eqs. (34) and (32). The covariance matrix for each mock catalogue is then

${ }^{(m)} \hat{C}_{i j}^{\mathrm{JK}}=\frac{N_{\mathrm{JK}}-1}{N_{\mathrm{JK}}} \sum_{k=1}^{N_{\mathrm{JK}}}\left(d_{i}^{k}-\bar{d}_{i}\right)\left(d_{j}^{k}-\bar{d}_{j}\right)$,

where the mean vector is obtained from the Jackknife samples

$\bar{d}_{j}=\frac{1}{N_{\mathrm{JK}}} \sum_{k=1}^{N_{\mathrm{JK}}} d_{i}^{k}$.

In addition, given our limited number of independent mock catalogue $N_{\mathrm{m}}$, we increase their number for lensing by resampling $N_{\mathrm{r}}$ times the observed lensing ellipticity distribution function, and the photometric redshifts distribution. We find this strategy to efficiently improve the accuracy of the covariance matrix for the lensing, especially at small scales. The final covariance matrix is therefore obtained by averaging the Jackknife covariance matrices

$\bar{C}_{i j}=\frac{1}{N_{\mathrm{m}} \times N_{\mathrm{r}}} \sum_{m=1}^{N_{\mathrm{m}} \times N_{\mathrm{r}}}{ }^{(m)} \hat{C}_{i j}^{J K}$.
Table 2. Properties of the simulated fields in terms of independent mock catalogue, random resampling of lensing shape noise and photometric redshifts per catalogue, and number of sub-regions for Jackknife resampling.

\begin{tabular}{lcccc}
\hline \hline Field name & Size (deg) & $\begin{array}{c}\text { Number } \\
\text { of mocks }\end{array}$ & $\begin{array}{c}\text { Number } \\
\text { of realizations }\end{array}$ & $\begin{array}{c}\text { Number of } \\
\text { subregions }\end{array}$ \\
\hline CS82 & $87.15 \times 2.58$ & 4 & 60 & 16 \\
W1 & $8.7 \times 6.3$ & 15 & 4 & 12 \\
W3 & $11.7 \times 6.6$ & 11 & 4 & 16 \\
W4 & $5.7 \times 5.6$ & 27 & 3 & 9 \\
\hline
\end{tabular}

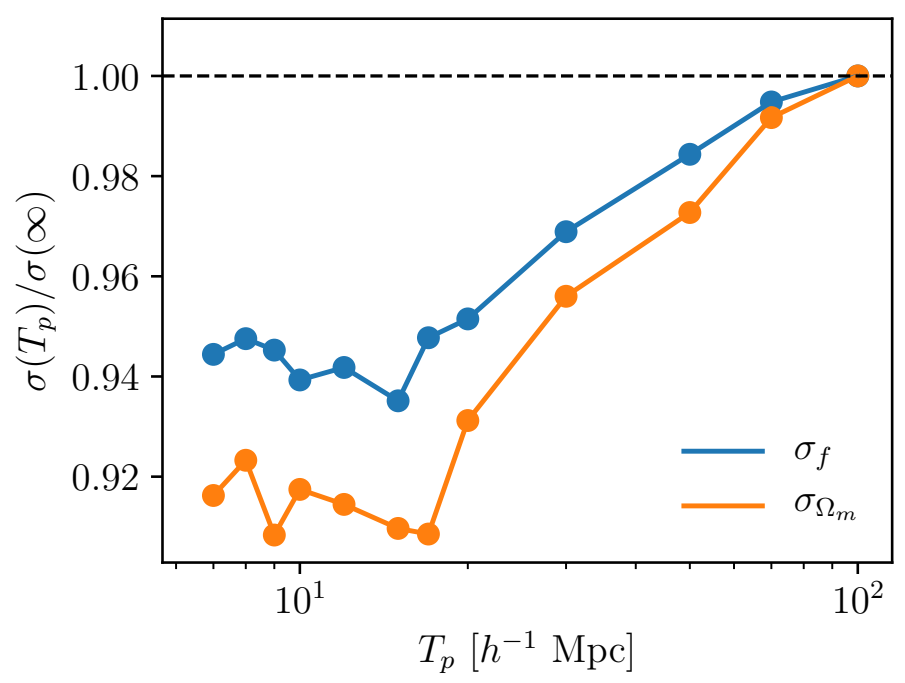

Fig. 6. Variation of the relative errors on the parameters $f$ and $\Omega_{\mathrm{m}}$, as a function of the smooth scale $T_{\mathrm{p}}$ in the covariance matrices. There is no improvement below $T_{\mathrm{p}}=12 \mathrm{~h}^{-1}$.

Finally, we computed the precision matrix

$\hat{\Phi}_{i j}=\left[\bar{C}_{i j}\right]^{-1}$.

Escoffier et al. (2016) have shown that this expression provides an unbiased estimate of the true precision matrix.

In spite of our resampling strategy, our covariance matrices are still noisy. Therefore, we adopted the tapering method proposed by Paz \& Sánchez (2015) to damp the noise by a filter function beyond a given tapering scale $T_{\mathrm{p}}$. This technique is based on the assumption that correlation between pairs of data points far apart is negligible and little information is lost by treating these points as being independent. Although very efficient, it is commonly accepted that this method might inadvertently remove non-Gaussian terms (Paz \& Sánchez 2015). However this effect is beyond the scope of this analysis given our data and the range of scales investigated in this work. In Fig. 6, we observe that large tapering yields errors similar to no tapering. In contrast, small tapering zeros all off-diagonal terms, and can also lead to overestimated errors. We find the errors on $f$ and $\Omega_{\mathrm{m}}$ to reach a minimum value at the tapering scale $T_{\mathrm{p}} \sim 12 \mathrm{~h}^{-1} \mathrm{Mpc}$. We adopted this scale in the rest of this analysis. We should note that all measurements were performed with $s_{\min }=14.1 h^{-1} \mathrm{Mpc}$ and $R_{0}=1.5 h^{-1} \mathrm{Mpc}$. However, we repeated some measurements with our final set-up $\left(s_{\min }=\right.$ $17.8 h^{-1} \mathrm{Mpc}$ and $R_{0}=1.0 h^{-1} \mathrm{Mpc}$ ) and find that these parameters have almost no impact on the tapering scale behaviour. The covariance and precision matrices obtained before and after tapering at this scale are shown in Fig. 7. We can observe that 

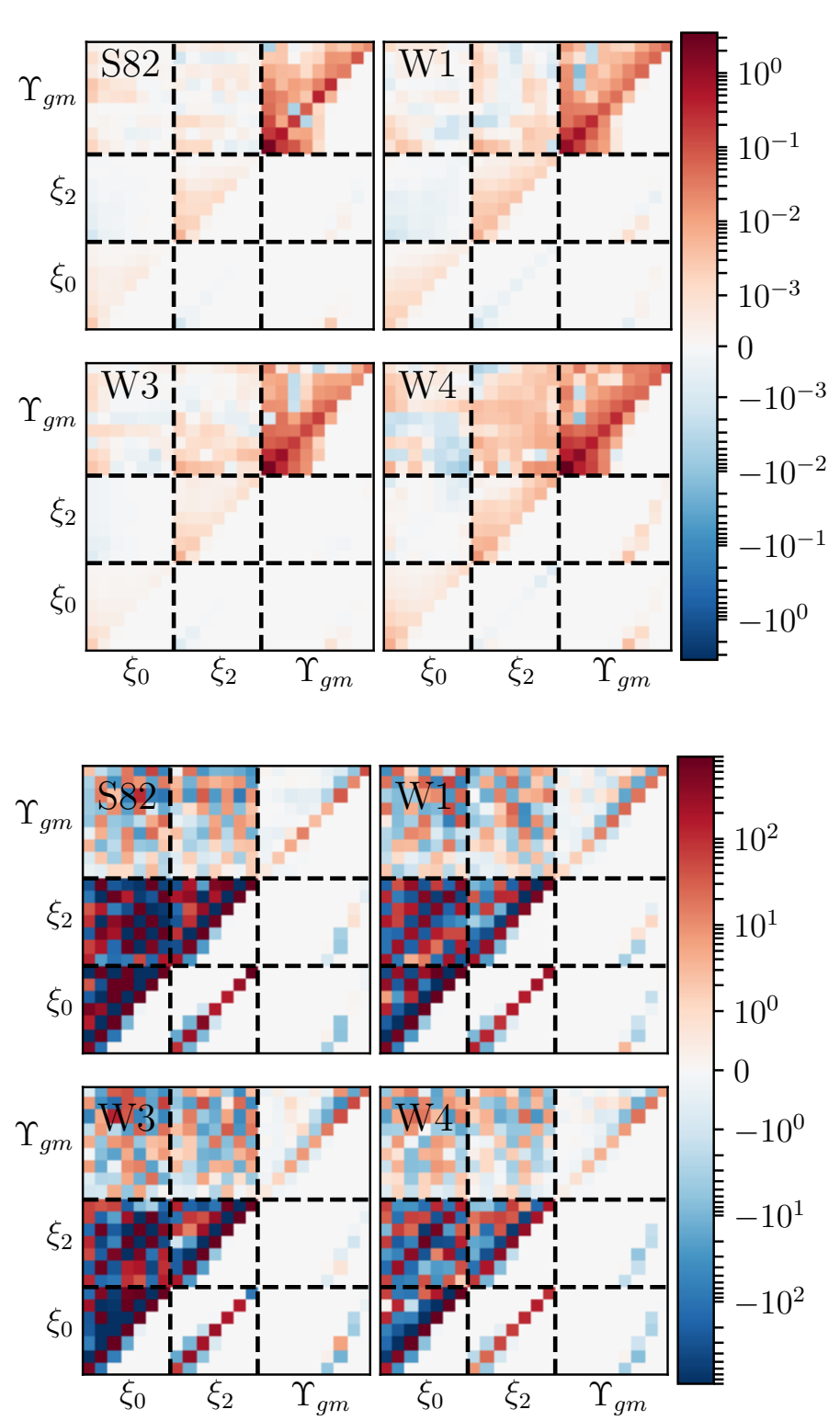

Fig. 7. Matrices of covariance (top panel) and precision (bottom panel) in logarithmic color scale for the 4 fields used in this analysis. In both panels, the upper triangular part of the matrices represents the case without tapering, while the lower part represents the case with tapering $T_{\mathrm{p}}=12 \mathrm{~h}^{-1} \mathrm{Mpc}$. Noise between far apart scales is significantly decreased and the errors on the model parameters converge to a minimum.

the noise in the off-diagonal terms is significantly reduced after tapering. This is particularly obvious between clustering and lensing, which cover very different range of scales.

\section{Cosmological results}

The quality tests and errors assessment that we performed with the simulations give us confidence that our dataset can lead to reliable cosmological constraints.

\subsection{Galaxy-clustering and galaxy-galaxy lensing measurements}

In Figs. 8 and 9, we show our RSD and GGL measurements, along with our theoretical predictions, assuming the fiducial

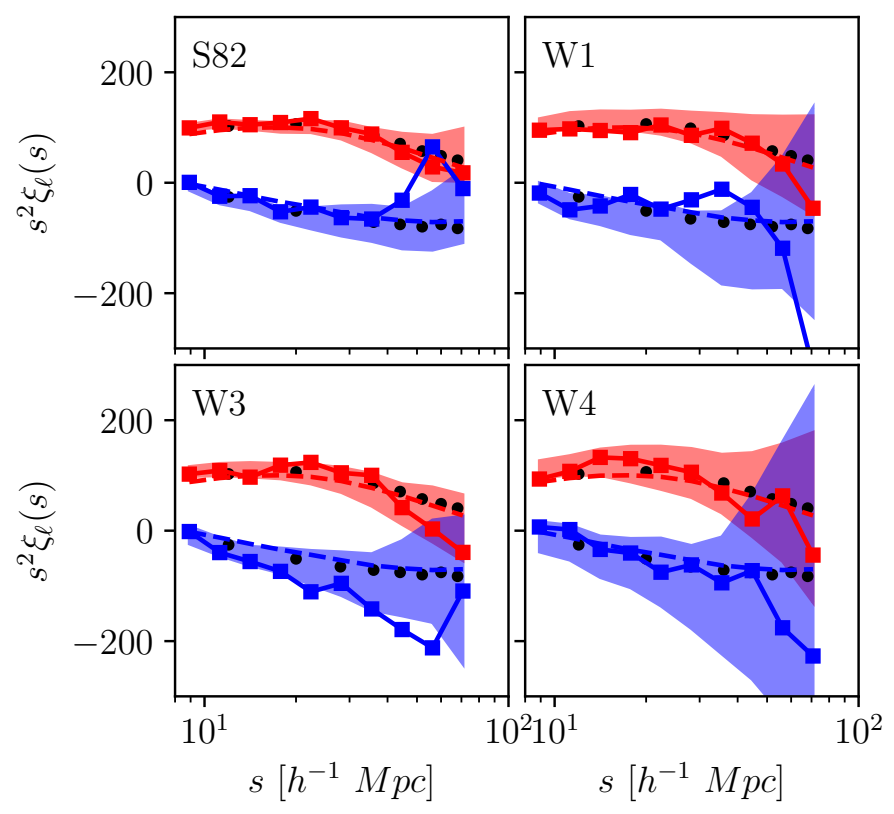

Fig. 8. Monopole (red) and quadrupole (blue) measurements with mock catalogues (shaded region), real data (solid lines), and theoretical predictions with a linear bias parameter $b_{1}=1.8$ (dashed lines). Black dots represent pre-reconstruction measurements with the full DR12v5 CMASS sample from Cuesta et al. (2016).

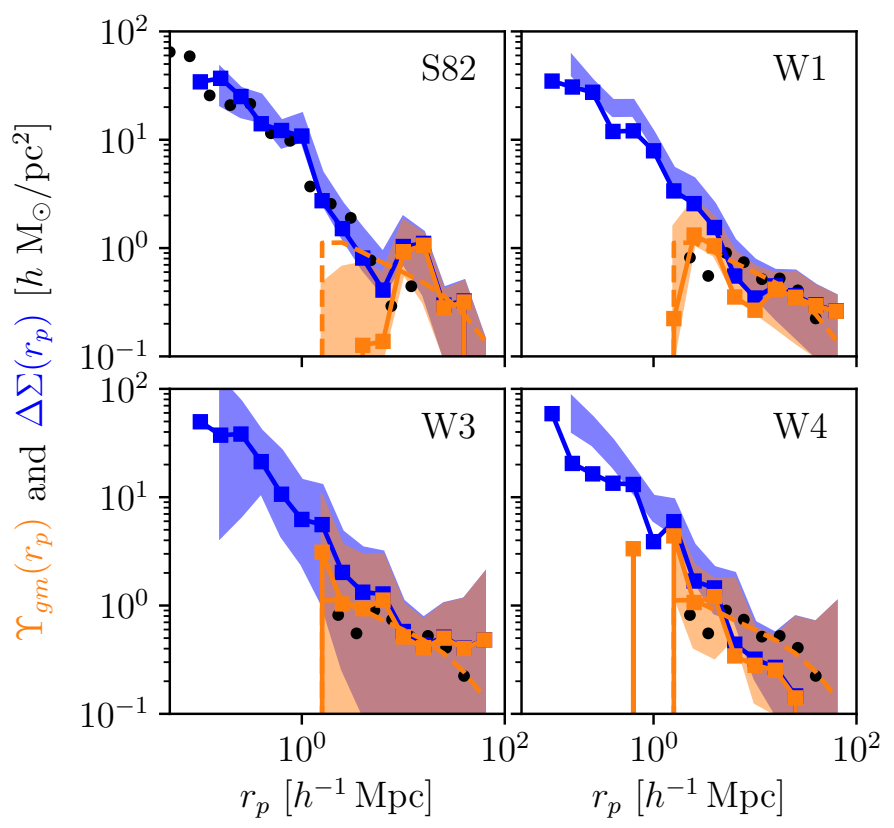

Fig. 9. Filtered $\Upsilon_{g m}$ and non-filtered $\Delta \Sigma$ GGL measurements with mocks (shaded regions), $\Delta \Sigma$ and $\Upsilon$ data (blue and orange points respectively), and theory with a linear bias parameter $b_{1}=1.8$ (dashed line). Black dots in S82 panel represent $\Delta \Sigma$ measurements from L16, and $\Upsilon_{\mathrm{gm}}$ measurements from Alam et al. (2017) in CFHTLS panels.

parameters of the simulation, and a constant linear bias $b_{1}=1.8$. We find a good agreement within $1 \sigma$ C.L. between mocks, data, and theoretical predictions for all fields. We notice that the quadrupole of the correlation function measurement in the field W3 is lower than the $1 \sigma$ C.L., and that the GGL measurement in the field W4 is lower than $1 \sigma$ C.L. at scales $R<1 h^{-1} \mathrm{Mpc}$. For field W3, we found that setting $\sigma_{8}(z=0.57)=0.9$ and $b_{1}=1.5$ could reconcile predictions with measurements, thus suggesting a sample variance effect. These values are within the 


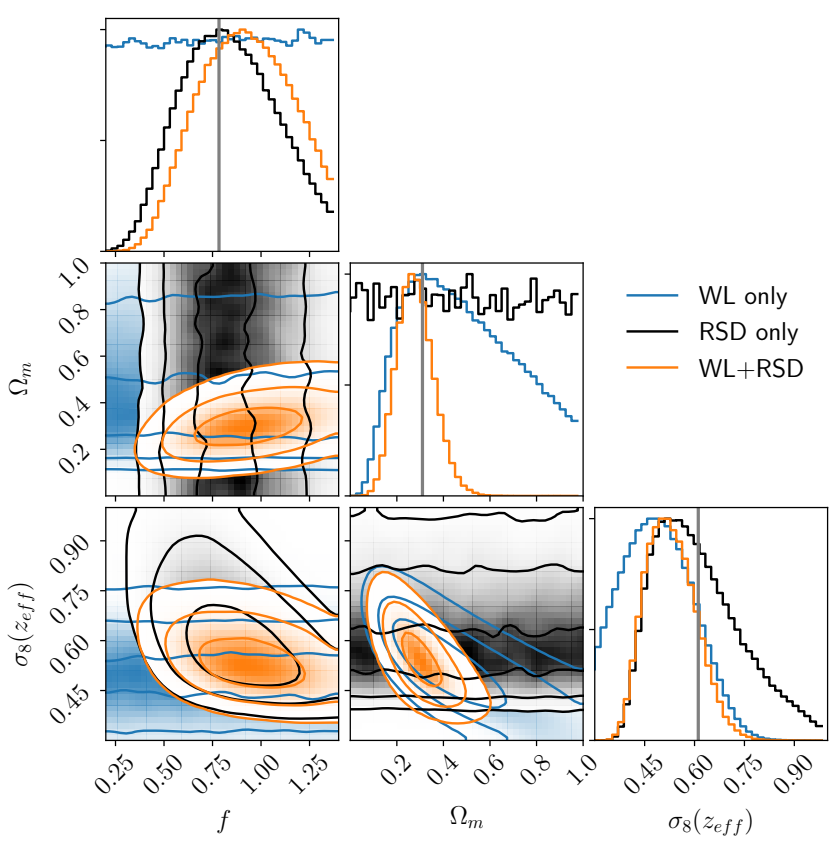

Fig. 10. Improvement on estimating $\Omega_{\mathrm{m}}$ and $\sigma_{8}$ when combining RSD and WL measurements. The blue and black curves are, respectively, obtained with WL and RSD constraints only. The orange curves are obtained with the combination of WL and RSD. Contours are given at 1, 2, and $3 \sigma$ C.L. Vertical lines indicate Planck TT,TE,EE+lowE 2018 results.

$3 \sigma$ C.L. of the RSD-only fit of the data (see Fig. 10). For field $\mathrm{W} 4$, we attribute the discrepancy to our poor modelling of baryonic or lensing effects at small scales, which average out too slowly in the data to reproduce the simulated dark-matter only profile. Nonetheless, the overall good agreement gives us confidence that we can proceed with the cosmological analysis.

\subsection{Growth of structure and background constraints}

We estimate the cosmological parameters $f, \sigma_{8}$, and $\Omega_{\mathrm{m}}$ by combining $\xi_{0}, \xi_{2}$, and $\Upsilon_{\mathrm{gm}}$ measurements. The power of this combination to break the degeneracy between $f$ and $\sigma_{8}$ has already been demonstrated (see e.g. DLT17, Joudaki et al. 2017). In this paper, we move one step further by estimating $\Omega_{\mathrm{m}}$ as well from the data. Figure 10 shows the independent lensing, clustering, and combined constraints on these parameters. Best-fit values and $1 \sigma$ error estimates are reported in Table 3. A corner plot with all the parameters involved in the fit is reported in Fig. A.2. On the one hand, we find that GGL alone constrains $\Omega_{\mathrm{m}}$ at $45 \%$ and $\sigma_{8}$ at $22 \%$. It provides no constraint on the structure growth rate $f$. On the other hand, RSD also constrains $\sigma_{8}$ at $20 \%$ but leaves $\Omega_{\mathrm{m}}$ completely unconstrained as expected from the model. When used in combination, GGL and RSD measurements yield $12 \%$ precision constraint on $\sigma_{8}$, i.e. almost as if the two datasets were independent. In fact, Fig. 10 shows that the well-known WL degeneracy between $\Omega_{\mathrm{m}}$ and $\sigma_{8}$ intersects almost perpendicularly with the constraint on $\sigma_{8}$ from RSD.

In Fig. 11, we present our estimate of the growth rate $f$, and compare to other measurements. In spite of having a wider area, we obtain a constraint similar to that found in DLT17 with VIPERS. Clearly, the number of RSD tracers determines the precision. In both analysis, we have about 28,000 galaxies in the range $0.5<z<0.7$. Regarding WL, the number densities of background sources at $z>0.7$ in both analysis are similar. We

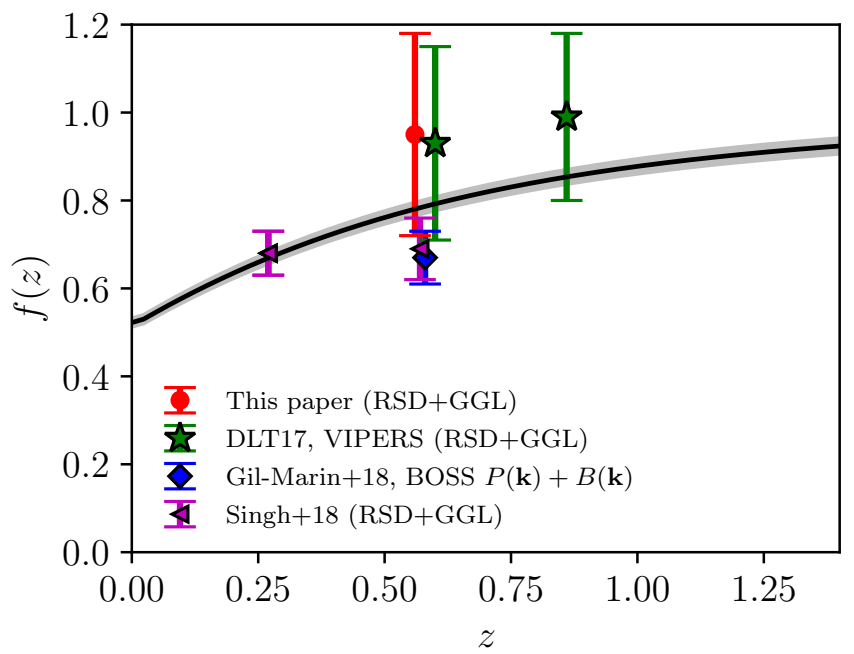

Fig. 11. Growth rate $f$ as a function of redshift compared to recent measurements. The black line and surrounding grey shared area indicate the Planck TT,TE,EE+lowE 2018 mean and $1 \sigma$ uncertainty predictions for $\Lambda \mathrm{CDM}-\mathrm{GR}$ flat model.

Table 3. Best-fit and derived parameters obtained by fitting the RSD only, GGL only, and their combination.

\begin{tabular}{lccc}
\hline \hline Parameters & RSD only & GGL only & GGL+RSD \\
\hline$\alpha$ & $1.02 \pm 0.05$ & $1.01 \pm 0.05$ & $1.02 \pm 0.05$ \\
$\epsilon$ & $0.01 \pm 0.05$ & $-0.01 \pm 0.06$ & $0.00 \pm 0.05$ \\
$f(z=0.57)$ & $0.86 \pm 0.24$ & - & $0.95 \pm 0.23$ \\
$\sigma_{8}(z=0.57)$ & $0.63 \pm 0.13$ & $0.50 \pm 0.11$ & $0.55 \pm 0.07$ \\
$\Omega_{\mathrm{m}}$ & - & $0.51 \pm 0.23$ & $0.31 \pm 0.08$ \\
\hline$b_{1}$ & $2.09 \pm 0.43$ & $1.94 \pm 0.55$ & $2.33 \pm 0.33$ \\
$b_{2}$ & $-0.06 \pm 0.53$ & $0.03 \pm 0.54$ & $-0.05 \pm 0.53$ \\
$\sigma_{v}\left(h^{-1} \mathrm{Mpc}\right)$ & $4.55 \pm 1.68$ & - & $4.20 \pm 1.64$ \\
\hline$S_{8}=\sigma_{8} \sqrt{\Omega_{\mathrm{m}} / 0.3}$ & - & $0.87 \pm 0.18$ & $0.72 \pm 0.08$ \\
$\widehat{E}_{\mathrm{G}}$ & - & - & $0.33 \pm 0.10$ \\
$f \sigma_{8}(z=0.57)$ & $0.53 \pm 0.14$ & $0.50 \pm 0.86$ & $0.51 \pm 0.12$ \\
$\sigma_{8}(z=0)$ & $0.78 \pm 0.26$ & $0.70 \pm 0.12$ & $0.73 \pm 0.08$ \\
\hline
\end{tabular}

have $n_{\mathrm{eff}}=3.45$ in CFHTLS fields and $n_{\mathrm{eff}}=2.33$ in CS82 and CFHTLS fields combined.

We also compare our results with analyses performed on the full CMASS sample. Singh et al. (2019) performed a joint analysis with Planck cosmic microwave background lensing and SDSS galaxy lensing and obtained three times tighter constraints than ours. Their results are in agreement with ours at the $1 \sigma$ C.L.

Finally, combining CMASS power spectrum and bispectrum, Gil-Marín et al. (2017) also obtained very competitive constraints at redshift $z=0.57$ in agreement with ours. These two estimates find a tension on $f$ with Planck predictions at $z=0.57$. Interestingly, this tension was also observed in other RSD analysis with the CMASS sample, but not with the LOWZ sample (e.g. Alam et al. 2017; Beutler et al. 2014).

\subsection{Comparison with other measurements}

From MCMC, we can derive new parameter constraints, defined as a combination of single parameters. In particular, we look at the quantity $S_{8}=\sigma_{8} \sqrt{\Omega_{\mathrm{m}} / 0.3}$, very common in gravitational lensing analyses. We find $S_{8}=0.72 \pm 0.08$, which is in 
agreement with the value estimated in L17, but $2-3 \sigma$ smaller than the cosmic microwave background measurements $S_{8}=$ $0.832 \pm 0.013$ (Planck Collaboration VI 2018). Similarly, our estimate of $\sigma_{8}=0.73 \pm 0.08$ is $2-3 \sigma$ smaller than the measurement $\sigma_{8}=0.8111 \pm 0.0060$ from the Planck collaboration 2018 . Our results are also in agreement with KIDS shear peaks statistics $S_{8}=0.75 \pm 0.059$ (Martinet et al. 2018; Shan et al. 2018), KIDS tomographic WL $S_{8}=0.745 \pm 0.039$ (Hildebrandt et al. 2017), and DES cosmological constraints from WL and clustering $S_{8}=0.783_{-0.025}^{+0.021}$. We note that our fit only performed with RSD measurements yield an estimate of $\sigma_{8}=0.78 \pm 0.26$, in better agreement with Planck estimates.

The linear galaxy bias parameter is known to be degenerate with the cosmological parameters $\Omega_{\mathrm{m}}$ and $\sigma_{8}$. In our fitting procedure, we assume a uniform prior on $b_{1}$ between 1 and 3, which largely encompasses the expected value for the CMASS sample. In their clustering analyses, Gil-Marín et al. (2017) found $b_{1} \sigma_{8}(z=0.57)=1.237 \pm 0.011$, and Chuang et al. (2013) found $b_{1} \sigma_{8}(z=0.57)=1.18 \pm 0.14$. We find $b_{1} \sigma_{8}(z=$ $0.57)=1.256 \pm 0.097$ in full agreement with these previous measurements. Marginalizing over $\sigma_{8}$, we find $b_{1}=2.33 \pm 0.33$, in agreement with White et al. (2011) and subsequent analyses (e.g. Ho et al. 2012; Nuza et al. 2013; Rodríguez-Torres et al. 2016).

Our model also contains a second order biasing term, but our estimated value $b_{2}=-0.04 \pm 0.53$ is not sufficient to discuss the non-linearity of the CMASS sample. We note that Gil-Marín et al. (2017) found $b_{2}=0.606 \pm 0.069$, which is in agreement with us.

Finally, we also include Alcock-Paczynski effect in our model, but found no significant constraint given the data, $\alpha=$ $1.01 \pm 0.05$ and $\epsilon=0.00 \pm 0.05$. We note that no significant constraint could either be obtained by Gil-Marín et al. (2017) with the full CMASS DR12 sample.

To conclude, we demonstrated the effectiveness of combining RSD and GGL to break the degeneracies between the amplitude of the large-scale structure fluctuations $\sigma_{8}$ and their growth rate $f$ at redshift $z=0.57$. We also found that the constraints on the cosmic matter density $\Omega_{\mathrm{m}}$, usually derived with WL, could be significantly improved by combining with RSD. Given the data, our measurements are still in agreement with Planck predictions.

\subsection{Measuring $E_{G}$}

To corroborate the information obtained with the analysis in the previous section and probe any deviation to $\Lambda$ CDM-GR predictions, we estimate $E_{\mathrm{G}}$, as defined in Reyes et al. (2010). The $E_{\mathrm{G}}$ estimator is function of projected scale $r_{\mathrm{p}}$, and is defined as (Zhang et al. 2007)

$E_{\mathrm{G}}\left(r_{\mathrm{p}}\right)=\frac{1}{\beta} \frac{\Upsilon_{\mathrm{gm}}\left(r_{\mathrm{p}}\right)}{\Upsilon_{\mathrm{gg}}\left(r_{\mathrm{p}}\right)}$.

This estimator is particularly interesting because it apparently just relies on observations. However, we show in the following that this might not be the case in practice.

Indeed, the $E_{\mathrm{G}}$ estimator suffers from a few downsides. First, this estimator relies on a previous determination of $\beta$. However, statistical and systematic error propagation into $E_{\mathrm{G}}$ error might be awkward, unless proper correction terms and covariance matrices are determined from ad hoc mock catalogues of lensing and clustering. Although seldom the case in the past, this is becoming more and more common (Blake et al. 2016; Amon et al. 2018; Singh et al. 2019).

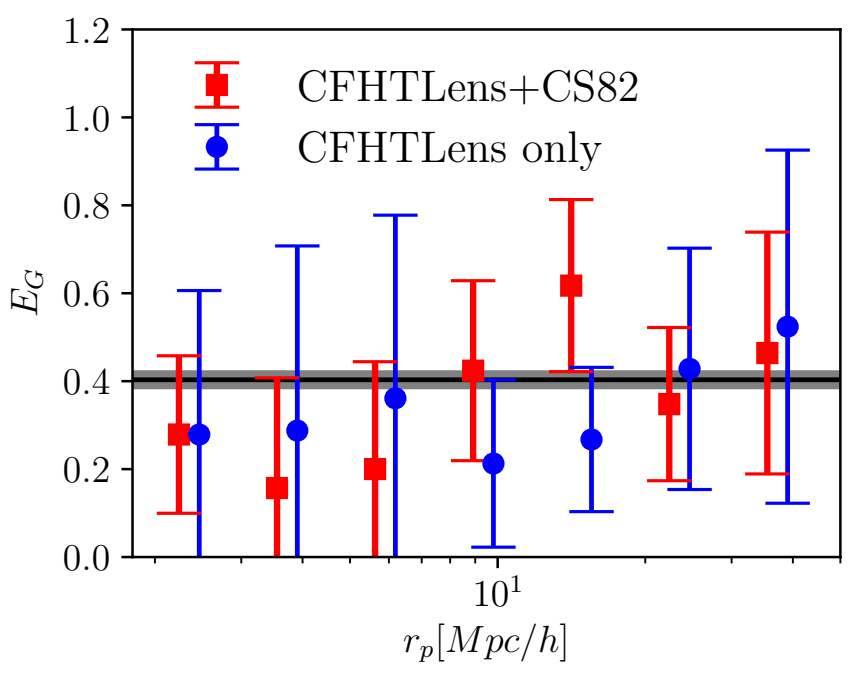

Fig. 12. Measurement of $E_{\mathrm{G}}$ with combined constraints in the fields CFHT-Stripe 82 and CFHTLs W1, W3, and W4. The horizontal black line indicates the Planck TT,TE,EE+lowE 2018 prediction. We note that CFHT-S82 data help shrink the error bars by about 30\%. CFHTLens points have been shifted rightwards for clarity.

Second, it is assumed that galaxy bias is linear, scaleindependent, and the galaxy density field is fully correlated to the underlying matter density field, i.e. the cross-correlation factor $r_{\mathrm{cc}}=1$. Of course, these assumptions hold in the linear regime, but the scale at which they break depends on the galaxy sample. Using CMASS mock catalogues, several authors have shown that they hold in the range $5<r_{\mathrm{p}}<60 \mathrm{~h}^{-1} \mathrm{Mpc}$ (Baldauf et al. 2010; White et al. 2011; Amon et al. 2018; Singh et al. 2019). This depends on the requested precision on the model though, and recent works have proposed to take non-linearity and other effects into account with normalizing functions derived from simulations (Alam et al. 2017; Singh et al. 2019). The multiplication of these correction terms nonetheless tend to reveal the limitation of the $E_{\mathrm{G}}$ estimator.

Marta Pinho et al. (2018) have noted that $E_{\mathrm{G}}$ depends not only on gravity but also on the background (e.g. quantified with the matter density $\Omega_{\mathrm{m} 0}$ in $\Lambda \mathrm{CDM}$ ). Although it is always possible to predict $E_{\mathrm{G}}$ for different cosmological models (see e.g. Zhang et al. 2007, in which predictions are computed for $\Lambda$ CDM, Flat DGP, $f(R)$ gravity, TeVeS/MOND), a discrepancy with the observations therefore does not specifically point to a failure of GR, but can also be attributed to the background. In this respect, these authors claim that an estimator such as $\eta$, based on independent estimates of $f \sigma_{8}(z), H(z), E_{\mathrm{G}}$ might be more appropriate. To our point of view, adjusting an actual model including modified gravity parameters might be as helpful.

In spite of these limitations, $E_{\mathrm{G}}$ has become quite popular recently, mostly because of the advent of wide field imaging and spectroscopic surveys. This estimator has been measured several times, but no significant deviation from $\Lambda \mathrm{CDM}-\mathrm{GR}$ has been found so far. In particular with the CMASS sample at redshift $z=0.57$, Amon et al. (2018) found $E_{\mathrm{G}}=0.26 \pm 0.08$, Blake et al. (2016) found $E_{\mathrm{G}}=0.30 \pm 0.07$, Pullen et al. (2016) found $E_{\mathrm{G}}=$ $0.24 \pm 0.06$, Alam et al. (2017) found $E_{\mathrm{G}}=0.42 \pm 0.06$, and Singh et al. (2019) found $E_{\mathrm{G}}=0.39 \pm 0.05$. The dispersion in the estimates reveal that the method is probably not fully mature yet, and deserves further investigation, in particular regarding the observational biases such as photometric redshifts.

Figure 12 shows our measurements of $E_{\mathrm{G}}$ as a function of scale. We estimate $\beta=0.41 \pm 0.15$ from our fit to the RSD 


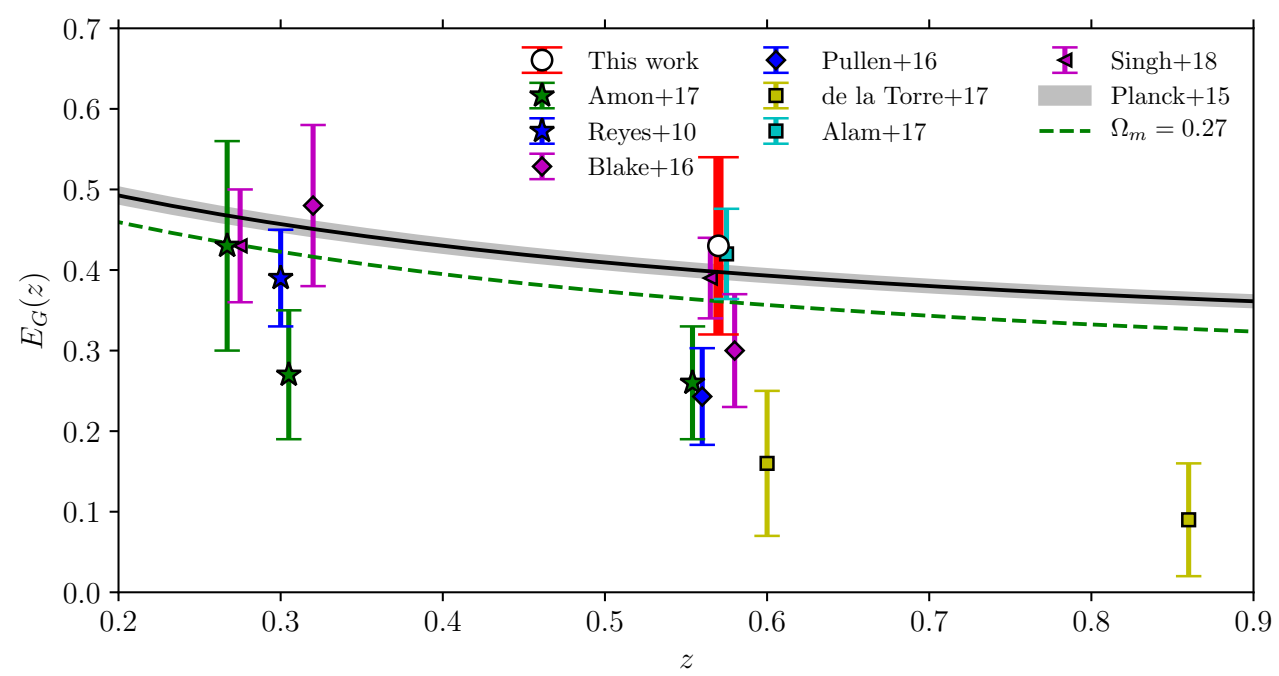

Fig. 13. Measurements of $E_{\mathrm{G}}$ at various redshifts. Effective redshifts of the measurements have been slightly shifted for clarity. measurements only. Although it is difficult to compare our work with other works because authors use different models, this value is larger but statistically consistent with that found with the full CMASS sample $\beta=0.34 \pm 0.02$ from Amon et al. (2018). For each bin of $E_{\mathrm{G}}\left(r_{p}\right)$, we add in quadrature the errors on the ratio $U^{i}=\Upsilon_{\mathrm{gm}}^{i} / \Upsilon_{\mathrm{gg}}^{i}$ derived from the data and the error on $\beta=f / b_{1}$ derived from the fit, with the following chain rule formula:

$\frac{\hat{C}\left(E_{\mathrm{G}}^{i}\right)}{E_{\mathrm{G}}^{i} E_{\mathrm{G}}^{i}}=\frac{\hat{C}\left(U^{i}\right)}{U^{i} U^{i}}+\frac{\hat{C}(\beta)}{\beta^{2}}+2 \sqrt{\frac{\hat{C}(\beta)}{\beta^{2}}} \sqrt{\frac{\hat{C}\left(U^{i}\right)}{U^{i} U^{i}}} \rho\left(\beta, U^{i}\right)$.

Using the MCMC samples from the fit of the GGL and RSD measurements, we use our model to reconstruct the ratio $U^{i}$. We also determine the correlation coefficients $\rho\left(\beta, U^{i}\right) \sim 0.3$, i.e. $\beta$ and $U^{i}$ are significantly correlated.

We average in the scale range $10<r_{\mathrm{p}}<60 h^{-1} \mathrm{Mpc}$, and we find $E_{\mathrm{G}}=0.48 \pm 0.15$ for CFHTLens field only, and $E_{\mathrm{G}}=0.43 \pm 0.11$ for CFHTLens and CS82 fields combined, i.e. a $30 \%$ improvement in precision for a $100 \%$ increase in area. In the average $E_{\mathrm{G}}$ calculation, we consider the full covariance matrix between the $E_{\mathrm{G}}$ points estimated from our simulations in Sect. 5.1. We note finally that our current precision does not justify applying scale-dependent bias, redshift weighting, or integration window corrections since their effect is less than $5 \%$ at the scales we consider (see Alam et al. 2017; Singh et al. 2019).

To put our measurement in context, we collected the $E_{\mathrm{G}}$ measurements at different redshifts from the literature in Fig. 13. Overall, we observe a trend of $E_{\mathrm{G}}$ values lower than predicted by Planck 2018. In the appendix, we forward model the $E_{\mathrm{G}}$ signal based on the MCMC samples output from the joint fit of the GGL and RSD measurements on mocks. Figure A.1 shows that the probability distribution function of the $E_{\mathrm{G}}$ estimator is skewed towards low values. Taking its mean value then necessarily leads to a biased-low estimation of $E_{\mathrm{G}}\left(r_{\mathrm{p}}\right)$. This result confirms the previous claim from Alam et al. (2017), and might also explain why so many $E_{\mathrm{G}}$ measurements are below the Planck 2018 predictions.

\section{Conclusions}

Understanding the current acceleration of the expansion of the Universe is one of the major goal of cosmology today. The combination of GGL and RSD is a remarkable avenue to distinguish the effect of gravity due to large-scale structures, and the effect of some scalar field on the background expansion rate.

In this work, we have demonstrated the power of this combination applied to the well-studied CMASS galaxy sample at the effective redshift $z=0.57$. Using a comprehensive set of lensing and galaxy mock catalogues, we investigated several sources of systematic biases and determined the confidence limits for our datasets. In particular, we found that thanks to spectroscopic data, we could correct the bias due to photometric redshift uncertainty for galaxies brighter than $i_{\mathrm{AB}}<22.5$, and $i_{\mathrm{AB}}<24$ in our CFHT-S82 and CFHTLens WL catalogues, respectively. These conservative magnitude cuts allow us to match our GGL measurements in the CFHT-S82 and CFHTLS fields, although at the cost of drastically reducing the number of WL sources. This conclusive remark highlights the crucial need of spectroscopic redshifts to calibrate the photometric redshift faint galaxies.

Building on this encouraging result, we pursue a cosmological analysis of the combined dataset. Thanks to the joint GGL and RSD constraints, we efficiently break the degeneracy between galaxy bias $b_{1}$, matter density $\Omega_{\mathrm{m}}$, matter power spectrum amplitude $\sigma_{8}$, and the structure growth rate $f$ at $z=0.57$. We find astrophysical CMASS parameters and cosmological parameters in agreement with measurements previously obtained by other authors (White et al. 2011; Beutler et al. 2014; Chuang et al. 2013; Gil-Marín et al. 2017; Joudaki et al. 2017) and with Planck 2018 predictions in the frame of the $\Lambda$ CDM-GR model.

Finally, we combine GGL and RSD measurements to estimate $E_{\mathrm{G}}$. By averaging in the range of scales $10<r_{\mathrm{p}}<$ $60 h^{-1} \mathrm{Mpc}$, we find $E_{\mathrm{G}}(z=0.57)=0.43 \pm 0.11$, which is in perfect agreement with Planck 2018 prediction $E_{\mathrm{G}}=0.40$. Also, we use our mocks to characterize the statistical properties of $E_{\mathrm{G}}$, and find that it has an asymmetric probability distribution, which tends to underestimate its mean value. This might explain part of the low values found in previous analysis. We also find that the reconstructed value of $E_{\mathrm{G}}=\Omega_{\mathrm{m} 0} / f$ derived from the fit of the GGL and RSD measurements results in a value with smaller errors bars than that obtained directly from the data. More importantly, this value naturally includes the cross-correlation terms between $\beta$ and $\Upsilon_{\mathrm{gg}}$.

Back in 2012, Gaztañaga et al. (2012) was already advocating that overlapping lensing and spectroscopic surveys were 100 times more constraining on the dark energy equation of state and cosmic growth history parameter $\gamma$. Although it might not be the 
cleanest way to test gravity, the recent progress in estimating $E_{\mathrm{G}}$ at different redshifts with different tracers comes as a confirmation. In the future, wider imaging and spectroscopic surveys will result in very tight constraints on cosmological parameters. In contrast, it will probably take us more time to fully profit from smaller but deeper imaging surveys. Deep imaging surveys are helpful for many reasons, but also introduce additional systematic errors on the lensing side, in particular with respect to blending (Harnois-Déraps et al. 2018; Euclid Collaboration 2019). Nonetheless, both strategies lead to very exciting perspectives regarding our understanding of the dark sector.

Acknowledgements. Based on observations obtained with MegaPrime/ MegaCam, a joint project of CFHT and CEA/DAPNIA, at the Canada-FranceHawaii Telescope (CFHT), which is operated by the National Research Council (NRC) of Canada, the Institut National des Science de l'Univers of the Centre National de la Recherche Scientifique (CNRS) of France, and the University of Hawaii. The Brazilian partnership on CFHT is managed by the Laboratório Nacional de Astrofísica (LNA). We appreciate the support of the Laboratório Interinstitucional de e-Astronomia (LIneA). We thank the CFHTLenS team. Funding for SDSS-III has been provided by the Alfred P. Sloan Foundation, the Participating Institutions, the National Science Foundation, and the US Department of Energy Office of Science. The SDSSIII web site is http://www.sdss3.org/. SDSS-III is managed by the Astrophysical Research Consortium for the Participating Institutions of the SDSS-III Collaboration including the University of Arizona, the Brazilian Participation Group, Brookhaven National Laboratory, Carnegie Mellon University, University of Florida, the French Participation Group, the German Participation Group, Harvard University, the Instituto de Astrofisica de Canarias, the Michigan State/Notre Dame/JINA Participation Group, Johns Hopkins University, Lawrence Berkeley National Laboratory, Max Planck Institute for Astrophysics, Max Planck Institute for Extraterrestrial Physics, New Mexico State University, New York University, Ohio State University, Pennsylvania State University, University of Portsmouth, Princeton University, the Spanish Participation Group, University of Tokyo, University of Utah, Vanderbilt University, University of Virginia, University of Washington, and Yale University. The BigMDPL simulation has been performed on the SuperMUC supercomputer at the LeibnizRechenzentrum (LRZ) in Munich, using the computing resources awarded to the PRACE project number 2012060963. We thank the Red Española de Supercomputación for granting us computing time in the Marenostrum Supercomputer a the BSC-CNS where part of the analyses presented in this paper have been performed. We appreciate the support of the OCEVU Labex (Grant $\mathrm{N}^{\circ}$ ANR-11 LABX-0060) and the A*MIDEX project (Grant No ANR-11-IDEX-0001-02) funded by the Investissements d'Avenir French government programme managed by the ANR. We also acknowledge support from the ANR eBOSS project (ANR-16-CE31-0021) of the French National Research Agency. CG acknowledges support from Centre National d'Etudes Spatiales, Italian Ministry of For eign Affairs and International Cooperation Directorate General for Country Promotion (Project "Crack the lens?"), from the agreement ASI n.I/023/12/0 "Attività relative alla fase B2/C per la missione Euclid", and from the Italian Ministry for Education, University and Research (MIUR) through the SIR individual grant SIMCODE (project number RBSI14P4IH). JPK acknowledges support from the "Cosmology with 3D-Maps of the Universe" SNF grant \#175751. GY acknowledges financial support from MINECO/FEDER under project gran AYA2015-63810-P

\section{References}

Aihara, H., Arimoto, N., Armstrong, R., et al. 2018, PASJ, 70, S4

Alam, S., Albareti, F. D., Allende Prieto, C., et al. 2015, ApJS, 219, 12

Alam, S., Miyatake, H., More, S., Ho, S., \& Mandelbaum, R. 2017, MNRAS, 465,4853

Alcock, C., \& Paczynski, B. 1979, Nature, 281, 358

Amendola, L., Kunz, M., \& Sapone, D. 2008, JCAP, 2008, 013

Amendola, L., Kunz, M., Motta, M., Saltas, I. D., \& Sawicki, I. 2013, Phys. Rev. D, 87, 023501

Amon, A., Blake, C., Heymans, C., et al. 2018, MNRAS, 479, 3422

Anderson, L., Aubourg, E., Bailey, S., et al. 2012, MNRAS, 427, 3435

Annis, J., Soares-Santos, M., Strauss, M. A., et al. 2014, ApJ, 794, 120

Baldauf, T., Smith, R. E., Seljak, U., \& Mandelbaum, R. 2010, Phys. Rev. D, 81, 063531

Baldauf, T., Seljak, U., Desjacques, V., \& McDonald, P. 2012, Phys. Rev. D, 86, 083540

Ballinger, W. E., Peacock, J. A., \& Heavens, A. F. 1996, MNRAS, 282, 877
Bean, R., Bernat, D., Pogosian, L., Silvestri, A., \& Trodden, M. 2007, Phys. Rev. D, 75, 064020

Behroozi, P. S., Wechsler, R. H., \& Wu, H.-Y. 2013, ApJ, 762, 109

Bekenstein, J. D. 2004, Phys. Rev. D, 70, 083509

Bel, J., Pezzotta, A., Carbone, C., Sefusatti, E., \& Guzzo, L. 2019, A\&A, 622, A109

Benítez, N. 2000, ApJ, 536, 571

Betoule, M., Kessler, R., Guy, J., et al. 2014, A\&A, 568, A22

Beutler, F., Saito, S., Seo, H.-J., et al. 2014, MNRAS, 443, 1065

Blake, C., Glazebrook, K., Davis, T. M., et al. 2011, MNRAS, 418, 1725

Blake, C., Joudaki, S., Heymans, C., et al. 2016, MNRAS, 456, 2806

Bolton, A. S., Schlegel, D. J., Aubourg, É., et al. 2012, AJ, 144, 144

Bundy, K., Leauthaud, A., Saito, S., et al. 2015, ApJS, 221, 15

Carbone, C., Petkova, M., \& Dolag, K. 2016, JCAP, 7, 034

Chuang, C.-H., \& Wang, Y. 2012, MNRAS, 426, 226

Chuang, C.-H., Prada, F., Cuesta, A. J., et al. 2013, MNRAS, 433, 3559

Coil, A. L., Blanton, M. R., Burles, S. M., et al. 2011, ApJ, 741, 8

Cuesta, A. J., Vargas-Magaña, M., Beutler, F., et al. 2016, MNRAS, 457, 1770

Damour, T. 2000, Nucl. Phys. B Proc. Suppl., 80, 41

Dark Energy Survey Collaboration 2005, ArXiv e-prints

[arXiv:astro-ph/0510346]

Dawson, K. S., Schlegel, D. J., Ahn, C. P., et al. 2013, AJ, 145, 10

de la Torre, S., \& Guzzo, L. 2012, MNRAS, 427, 327

de la Torre, S., Guzzo, L., Peacock, J. A., et al. 2013, A\&A, 557, A54

de la Torre, S., Jullo, E., Giocoli, C., et al. 2017, A\&A, 608, A44

DES Collaboration (Abbott, T. M. C., et al.) 2018, Phys. Rev. D, 98, 043526

DESI Collaboration (Aghamousa, A., et al.) 2016a, ArXiv e-prints [arXiv:1611.00036]

DESI Collaboration (Aghamousa, A., et al.) 2016b, ArXiv e-prints [arXiv:1611.00037]

Dvali, G., Gabadadze, G., \& Porrati, M. 2000, Phys. Lett. B, 485, 208

Dvornik, A., Hoekstra, H., Kuijken, K., et al. 2018, MNRAS, 479, 1240

Eisenstein, D. J., Weinberg, D. H., Agol, E., et al. 2011, AJ, 142, 72

Erben, T., Hildebrandt, H., Miller, L., et al. 2013, MNRAS, 433, 2545

Escoffier, S., Cousinou, M. C., Tilquin, A., et al. 2016, ArXiv eprints[arXiv:1606.00233]

Euclid Collaboration (Martinet, N., et al.) 2019, A\&A, 627, A59

Feldman, H. A., Kaiser, N., \& Peacock, J. A. 1994, ApJ, 426, 23

Ferreira, P. G., \& Skordis, C. 2010, Phys. Rev. D, 81, 104020

Fisher, K. B., Davis, M., Strauss, M. A., Yahil, A., \& Huchra, J. P. 1994, MNRAS, 267, 927

Garilli, B., Le Fèvre, O., Guzzo, L., et al. 2008, A\&A, 486, 683

Gaztañaga, E., Eriksen, M., Crocce, M., et al. 2012, MNRAS, 422, 2904

Gil-Marín, H., Wagner, C., Noreña, J., Verde, L., \& Percival, W. 2014, JCAP, 12, 029

Gil-Marín, H., Percival, W. J., Verde, L., et al. 2017, MNRAS, 465, 1757

Giocoli, C., Jullo, E., Metcalf, R. B., et al. 2016, MNRAS, 461, 209

Gunn, J. E., Siegmund, W. A., Mannery, E. J., et al. 2006, AJ, 131, 2332

Guzzo, L., Scodeggio, M., Garilli, B., et al. 2014, A\&A, 566, A108

Harnois-Déraps, J., Amon, A., Choi, A., et al. 2018, MNRAS, 481, 1337

Heymans, C., Van Waerbeke, L., Miller, L., et al. 2012, MNRAS, 427, 146

Hildebrandt, H., Erben, T., Kuijken, K., et al. 2012, MNRAS, 421, 2355

Hildebrandt, H., Viola, M., Heymans, C., et al. 2017, MNRAS, 465, 1454

Ho, S., Cuesta, A., Seo, H.-J., et al. 2012, ApJ, 761, 14

Joudaki, S., Mead, A., Blake, C., et al. 2017, MNRAS, 471, 1259

Klypin, A., Yepes, G., Gottlöber, S., Prada, F., \& Heß, S. 2016, MNRAS, 457, 4340

Laureijs, R., Amiaux, J., Arduini, S., et al. 2011, ArXiv e-prints [arXiv:1110.3193]

Leauthaud, A., Saito, S., Hilbert, S., et al. 2017, MNRAS, 467, 3024

Leonard, C. D., Ferreira, P. G., \& Heymans, C. 2015, JCAP, 2015, 051

Lesgourgues, J. 2011, ArXiv e-prints [arXiv:1104 .2932]

Lima, M., Cunha, C. E., Oyaizu, H., et al. 2008, MNRAS, 390, 118

LSST Dark Energy Science Collaboration 2012, ArXiv e-prints [arXiv:1211.0310]

Magaña, J., Motta, V., Cárdenas, V. H., Verdugo, T., \& Jullo, E. 2015, ApJ, 813, 69

Mandelbaum, R., Hirata, C. M., Broderick, T., Seljak, U., \& Brinkmann, J. 2006 MNRAS, 370, 1008

Mandelbaum, R., Seljak, U., Hirata, C. M., et al. 2008, MNRAS, 386, 781

Maraston, C., Pforr, J., Henriques, B. M., et al. 2013, MNRAS, 435, 2764

Marta Pinho, A., Casas, S., \& Amendola, L. 2018, JCAP, 11, 027

Martinet, N., Schneider, P., Hildebrandt, H., et al. 2018, MNRAS, 474, 712

Matsubara, T., \& Suto, Y. 1996, ApJ, 470, L1

McDonald, P., \& Roy, A. 2009, JCAP, 8, 020

Metcalf, R. B., \& Petkova, M. 2014, MNRAS, 445, 1942

Moraes, B., Kneib, J.-P., Leauthaud, A., et al. 2014, Rev. Mex. Astron. Astrofis Conf. Ser., 44, 202 
E. Jullo et al.: Testing gravity from RSD and galaxy-galaxy lensing

Nakajima, R., Mandelbaum, R., Seljak, U., et al. 2012, MNRAS, 420, 3240

Newman, J. A., Cooper, M. C., Davis, M., et al. 2013, ApJS, 208, 5

Nuza, S. E., Sánchez, A. G., Prada, F., et al. 2013, MNRAS, 432, 743

Paz, D. J., \& Sánchez, A. G. 2015, MNRAS, 454, 4326

Pezzotta, A., de la Torre, S., Bel, J., et al. 2017, A\&A, 604, A33

Planck Collaboration XIII. 2016, A\&A, 594, A13

Planck Collaboration VI. 2018, ArXiv e-prints [arXiv:1807. 06209]

Pullen, A. R., Alam, S., He, S., \& Ho, S. 2016, MNRAS, 460, 4098

Reid, B., Ho, S., Padmanabhan, N., et al. 2016, MNRAS, 455, 1553

Reyes, R., Mandelbaum, R., Seljak, U., et al. 2010, Nature, 464, 256

Rodríguez-Torres, S. A., Chuang, C.-H., Prada, F., et al. 2016, MNRAS, 460, 1173

Ross, A. J., Percival, W. J., Sánchez, A. G., et al. 2012, MNRAS, 424, 564

Ross, A. J., Beutler, F., Chuang, C.-H., et al. 2017, MNRAS, 464, 1168

Saito, S., Baldauf, T., Vlah, Z., et al. 2014, Phys. Rev. D, 90, 123522

Seo, H.-J., \& Eisenstein, D. J. 2003, ApJ, 598, 720

Shan, H., Liu, X., Hildebrandt, H., et al. 2018, MNRAS, 474, 1116
Shirasaki, M., Takada, M., Miyatake, H., et al. 2017, MNRAS, 470, 3476 Simpson, F., Heymans, C., Parkinson, D., et al. 2013, MNRAS, 429, 2249 Singh, S., Mandelbaum, R., Seljak, U., Slosar, A., \& Vazquez Gonzalez, J. 2017, MNRAS, 471, 3827

Singh, S., Alam, S., Mandelbaum, R., et al. 2019, MNRAS, 482, 785

Smith, R. E., Peacock, J. A., Jenkins, A., et al. 2003, MNRAS, 341, 1311

Sugai, H., Karoji, H., Takato, N., et al. 2012, in Ground-based and Airborne Instrumentation for Astronomy IV, SPIE Conf. Ser., 8446, 84460Y

Takahashi, R., Sato, M., Nishimichi, T., Taruya, A., \& Oguri, M. 2012, ApJ, 761, 152

Taruya, A., Nishimichi, T., \& Saito, S. 2010, Phys. Rev. D, 82, 063522

Taylor, A., \& Joachimi, B. 2014, MNRAS, 442, 2728

Tully, R. B., Courtois, H. M., \& Sorce, J. G. 2016, AJ, 152, 50

White, M., Blanton, M., Bolton, A., et al. 2011, ApJ, 728, 126

Xu, X., Cuesta, A. J., Padmanabhan, N., Eisenstein, D. J., \& McBride, C. K. 2013, MNRAS, 431, 2834

Zhang, P., Liguori, M., Bean, R., \& Dodelson, S. 2007, Phys. Rev. Lett., 99, 141302 


\section{Appendix A: Weak-lensing systematics tests}

Masking. In order to assess the impact of missing tiles and large-scale masking (e.g. due to very bright stars), we compute the density of CS82 galaxies on a grid with pixel size $\sim 1 \mathrm{deg}$. Then, we randomly draw mock galaxies in the field such that the overall redshift distribution and total number of sources matches observations. Finally, we down-sample this catalogue according the density fluctuations attributed to masking. We find that masking increases the statistical noise in the GGL measurement by about $20 \%$ at all scales. However we could not identify any obvious systematic bias related to masking.

Photometric redshifts bias. Mandelbaum et al. (2008) and Nakajima et al. (2012) proposed an alternative method to estimate the bias introduced by photometric redshifts on GGL measurements. These authors proposed to estimate the bias $b_{z}\left(z_{\text {lens }}\right)$ between photometric redshifts $\Delta \tilde{\Sigma}$ and spectroscopic redshifts $\Delta \Sigma$ measurements,

$1+b_{z}\left(z_{\text {lens }}\right)=\frac{\Delta \tilde{\Sigma}}{\Delta \Sigma}=\frac{\sum_{j} w_{j} \Sigma_{\mathrm{cr}}^{-1} \tilde{\Sigma}_{\mathrm{cr}}^{-1}}{\sum_{j} w_{j} \tilde{\Sigma}_{\mathrm{cr}}^{-2}}$.

The summation is performed over the subset of source galaxies with both spectroscopic and photometric redshifts. We adapted the original expression from Mandelbaum et al. (2008) such that the inverse critical densities $\Sigma_{\mathrm{cr}}^{-1}=\frac{4 \pi G}{c^{2}} D_{\mathrm{L}}\left(1-\frac{D_{\mathrm{L}}}{D_{\mathrm{S}}}\right)$ converges to zero when the source redshift becomes smaller than $z_{\text {lens }}$. The value $w_{j}$ is the weight on source galaxy $j$ in the lensing catalogue. In order to estimate the effective bias on our GGL measurements with CMASS galaxies, we need to integrate the bias function $b_{z}\left(z_{\text {lens }}\right)$ over the CMASS redshift distribution $p\left(z_{\text {lens }}\right)$ such that

$\left\langle b_{z}\right\rangle=\frac{\int \mathrm{d} z_{\text {lens }} p\left(z_{\text {lens }}\right) \tilde{w}_{1}\left(z_{\text {lens }}\right) b_{z}\left(z_{\text {lens }}\right)}{\int \mathrm{d} z_{\text {lens }} p\left(z_{\text {lens }}\right) \tilde{w}_{1}\left(z_{\text {lens }}\right)}$,

where the weight on each lens place $\tilde{w}_{1}=D_{\mathrm{L}}^{-2}(1+$ $\left.z_{\text {lens }}\right)^{-2} \sum_{j} w_{j} \Sigma_{\mathrm{cr}}^{-2}$ is correcting for the fact that the number of sources involved in a given aperture in physical coordinates includes more objects at lower than at higher redshifts. We bootstrapped our catalogues to estimate the uncertainties on our bias estimates.

For this measurement, we used VVDS $\left(i_{\mathrm{AB}}<22.5\right.$, Garilli et al. 2008), DEEP2 $\left(R_{\mathrm{AB}}<24.1\right.$, Newman et al. 2013), PRIMUS $\left(i_{\mathrm{AB}}<23.5\right.$, Coil et al. 2011), VIPERS $\left(i_{\mathrm{AB}}<22.5\right.$, Guzzo et al. 2014), and SDSS-DR13 spectroscopic redshifts, which we matched to our lensing sources in our four fields. On Stripe 82, we obtained $b_{z}=-0.028 \pm 0.006, b_{z}=-0.131 \pm 0.004$ and $b_{z}=-0.082 \pm 0.004$ for BPZ, neural network or LePhare codes, respectively. With CFHTLens, we obtained $b_{z}=$ $+0.003 \pm 0.003, b_{z}=-0.014 \pm 0.004$ and $b_{z}=+0.022 \pm 0.003$ on fields $\mathrm{W} 1, \mathrm{~W} 3$, and $\mathrm{W} 4$, respectively.

We also adapted our code to assess the improvement obtained by using the photometric redshift probability of each source galaxy $p\left(z_{s}\right)$ instead of maximum-likelihood values. The critical densities then become

$\tilde{\Sigma}_{\mathrm{cr}}^{-1}=\frac{4 \pi G}{c^{2}} D_{\mathrm{L}} \int \mathrm{d} z_{\mathrm{s}} p\left(z_{\mathrm{s}}\right)\left(1-\frac{D_{\mathrm{L}}}{D_{\mathrm{S}}}\right)$.

On field Stripe 82, we found that using the full probability $p\left(z_{\mathrm{s}}\right)$ halved the bias obtained with LePhare code to

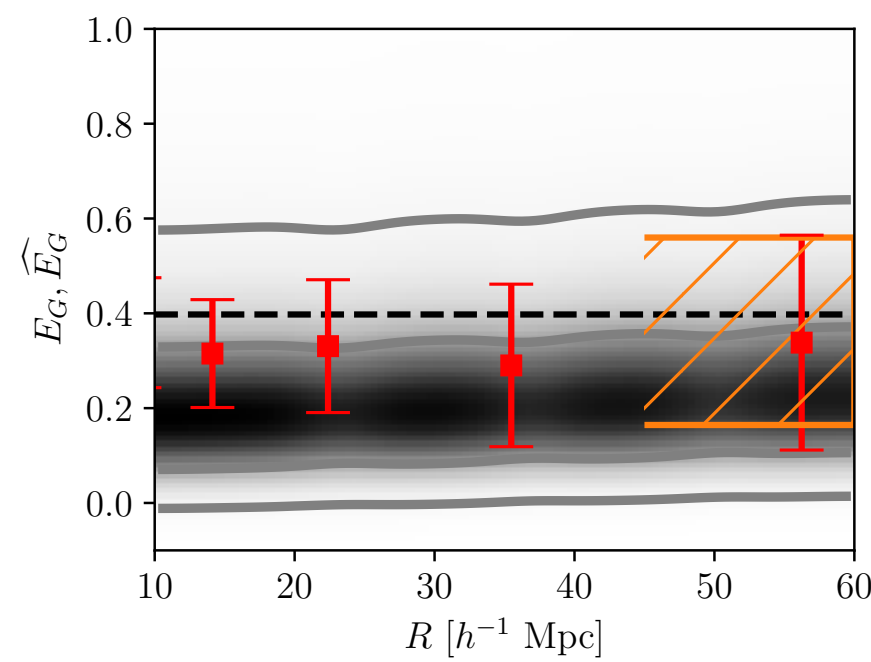

Fig. A.1. Recovered signal in the mocks, when $E_{\mathrm{G}}=\frac{\Upsilon_{\mathrm{gm}}}{\beta \Upsilon_{\mathrm{gg}}}$ (grey shared area with $1 \sigma$ and $2 \sigma$ C.L. contours) and $\widehat{E_{\mathrm{G}}}=\Omega_{\mathrm{m}} / f$ (orange $1 \sigma$ shaded area) are computed from the MCMC samples, and when $E_{\mathrm{G}}=\frac{\Upsilon_{\mathrm{gm}}}{\beta \Upsilon_{\mathrm{gg}}}$ is directly estimated from the mocks (red data points with $1 \sigma$ error bars). In this latter case, we take $\beta=0.84 / 2.13=0.39$, as obtained from a previous fit of our model to the RSD-only measurements in the mocks. Both definitions are in agreement with the value of $\Omega_{\mathrm{m}} / f$ computed using the Planck cosmology 2018 of the simulation (black dashed line). Measurements are performed with $R_{0}=1.0 h^{-1} \mathrm{Mpc}$, $s_{\min }=17.8 h^{-1} \mathrm{Mpc}$ and no tapering.

$b_{z}=-0.031 \pm 0.005$. Nonetheless, using the best-fit redshifts provided by BPZ still yields the smallest bias.

Catastrophic photometric redshifts. In order to assess the impact of catastrophic redshifts on the lensing measurements, we computed the two-dimensional probability $p\left(z_{\mathrm{LP}} \mid z_{\mathrm{ANNZ}}\right)$ of obtaining a photometric redshift with Le Phare given a photometric redshift obtained with neural network. Assuming this later to be the true redshift, we degraded the true redshifts in our mocks to reproduce the catastrophic outlier effects. Overall, we found that catastrophic redshifts could bias the lensing signal by about $b_{z}=+0.03$. This is in agreement with our estimations above with spectroscopic redshifts and the estimates found in Leauthaud et al. (2017).

Asymmetric posterior on. $E_{\mathrm{G}}$ It is very typical that observational estimators obtained from a ratio of observables have asymmetric probability distribution function. Indeed using our simulations, we found that $E_{\mathrm{G}}$ is systematically lower than $\Lambda \mathrm{CDM}-\mathrm{GR}$ predictions, with a long tail towards larger values of $E_{\mathrm{G}}$, as shown in Fig. A.1. When applying the usual $E_{\mathrm{G}}=\frac{\Upsilon_{\mathrm{gm}}}{\beta \Upsilon_{\mathrm{gg}}}$ estimator on our mocks, we also find mean values smaller than expected, although still in statistical agreement. We note finally that $1 \sigma$ constraints are tighter with the fit than with the usual $E_{\mathrm{G}}$ estimator. Given that Amendola et al. (2013) have demonstrated that $E_{\mathrm{G}}$ is probably not as effective as anticipated in some particular cosmological models, and Alam et al. (2017) and Singh et al. (2019) have started to apply bias corrections to this estimator based on mock catalogues built with fiducial cosmological models, we think it might be as efficient and clean to fit the correlation functions, and derive $E_{\mathrm{G}}$ by marginalizing over the model parameters, or simply compare $\Omega_{\mathrm{m} 0}$ and $f$ to their $\Lambda \mathrm{CDM}+\mathrm{GR}$ predictions. 
E. Jullo et al.: Testing gravity from RSD and galaxy-galaxy lensing

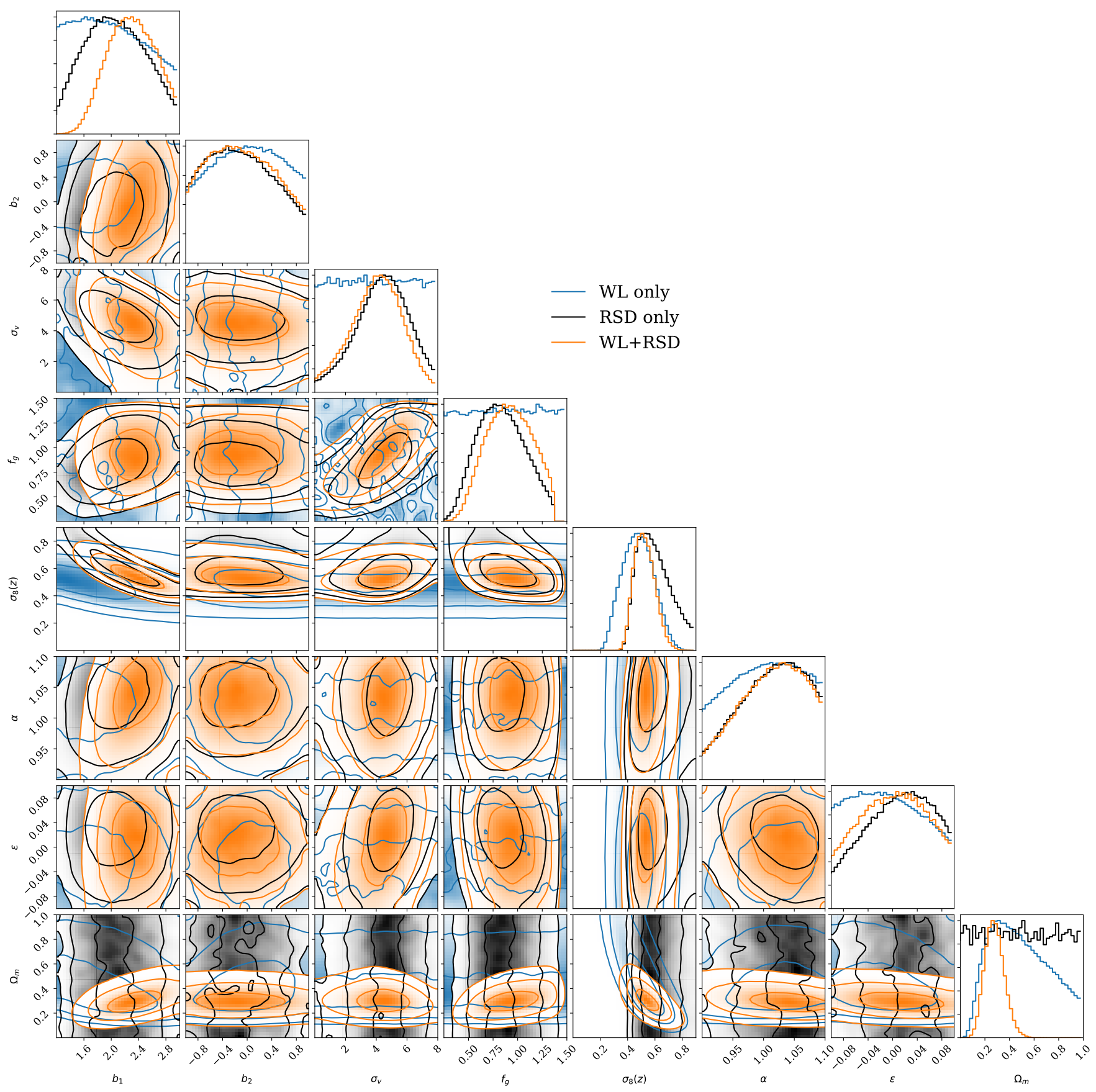

Fig. A.2. Contours at 1, 2, and $3 \sigma$ C.L. of all our model parameters estimated with the RSD only, GGL only, and their combination. In all cases, we set $s_{\min }=17.8 h^{-1} \mathrm{Mpc}$ and $R_{0}=1.0 h^{-1} \mathrm{Mpc}$ (see text for details). 